\title{
BOUNDARY BEHAVIOUR OF OPEN, LIGHT MAPPINGS IN METRIC MEASURE SPACES
}

\author{
Mihai Cristea \\ University of Bucharest, Faculty of Mathematics and Computer Sciences \\ Str. Academiei 14, R-010014, Bucharest, Romania; mcristea@fmi.unibuc.ro
}

\begin{abstract}
We study the boundary behaviour of open, light mappings satisfying generalized modular inequalities in general metric measure spaces. We extend in this way known facts from the theory of quasiregular mappings and from their recent generalizations, namely the mappings of finite distortion and the so called ring mappings.
\end{abstract}

\section{Introduction.}

A well-known, basic tool in the study of quasiregular mappings is the modular inequality of Poleckii

$$
M(f(\Gamma)) \leq K M(\Gamma)
$$

valid for all path families $\Gamma$ in $D$. Here $D$ is a domain in $\mathbf{R}^{n}$, the mapping $f: D \rightarrow$ $\mathbf{R}^{n}$ is quasiregular and $M$ is the modulus of a curve family (see the monographs $[45,46]$ and $[63,64]$ for more information about quasiregular mappings). Several generalizations of quasiregular mappings have been studied in the last 35 years. The most important is the class of mappings of finite distortion (see the monographs [27] and [31] and the papers [34, 44]). The classes of mappings distinguished by moduli inequalities and also defined on open sets in $\mathbf{R}^{n}$ were intensively studied in the last 15 years (see [10-13, 15-19, 29, 35, 39-41, 47-49, 52-57, 63]). Such an approach was proposed by Martio for homeomorphisms between open sets in $\mathbf{R}^{n}$ and the topic was summarized in the resulting monograph [41] written with Ryazanov, Srebro and Yakubov. Another generalization of this well-known class of quasiregular mappings is the class of quasiconformal maps on general metric measure spaces (see $[1,2,7,8$, 23-26, 32, 33]). Quasiregular mappings on metric measure spaces were studied in [6, 9, 14, 21, 22, 43]. Finally, homeomorphisms and open, discrete mappings satisfying generalized modular inequalities were studied in $[4,5,30,50,51,57-61,64]$ on generalized metric measure spaces, other then $\mathbf{R}^{n}$ with the euclidean metric. In [30] the boundary behaviour and equicontinuity of bounded open, discrete mappings on Riemannian manifolds for which a Poleckii type modular inequality holds is studied (see Theorem 5.4 in [30]). The same thing is studied on Ahlfors $Q$-regular metric measure spaces in [58] and on factor spaces in [60] and a Poleckii type modular inequality is given in [60]. We extend some of these results and some older results from the theory of quasiregular mappings from [38] and of the mappings of finite distortion from [10] on general metric measure spaces and for continuous, open, light mappings $f: X \rightarrow Y$.

In this paper $X, Y$ will be metric measure spaces endowed with Borel regular measures $\mu$ and $\nu$ such that $0<\mu(B)<\infty$ for every ball $B$ in $X$ and $0<\nu(B)<\infty$

https://doi.org/10.5186/aasfm.2021.4672

2020 Mathematics Subject Classification: Primary 30C65, 30L10.

Key words: Quasiregular mappings, generalization, boundary behaviour, open and light mappings, generalized modular inequalities, general metric measure spaces. 
for every ball $B$ in $Y$ and we suppose that the spaces $X$ and $Y$ are locally pathwise connected and locally compact and have countable bases of neighbourhoods. The distance on $X$ and $Y$ will be denoted by $d$. We also suppose that $X$ is proper (i.e. every closed ball $B \subset X$ is compact) and we denote $\bar{X}=X \cup\{\infty\}$ the Alexandrov compactification of $X$. In such a space if $\bar{X} \neq X$ and if $d\left(x, x_{n}\right) \rightarrow \infty$ for some $x \in X$, we say that $x_{n} \rightarrow \infty$. We work with continuous, open, light mappings $f: D \subset X \rightarrow Y$, where $D \subset X$ is open and $Y$ will always be Ahlfors $Q$-regular and will support a $(1, q)$-Poincaré inequality with $Q-1<q \leq Q$. Every Riemannian $n$-manifold is Ahlfors $n$-regular. The basic approach of the theory on metric measure spaces is from Väisälä [65]. We give the complete proof in Section 2, just for the sake of completeness.

Let $\gamma:[a, b] \rightarrow X$ be a path and let $\Delta=\left(a=t_{0}<t_{1}<, \ldots,<t_{n}=b\right)$ be a subdivision of $[a, b]$. Let $\mathcal{D}([a, b])$ be the set of all subdivisions of $[a, b]$. We set $V_{\Delta}(\gamma)=\sum_{i=1}^{n} d\left(\gamma\left(t_{i-1}\right), \gamma\left(t_{i}\right)\right)$ and $l(\gamma)=\sup _{\Delta \in \mathcal{D}([a, b])} V_{\Delta}(\gamma)$ be the length of $\gamma$. If $l(\gamma)<\infty$, we say that $\gamma$ is rectifiable. We also have $l(\gamma)=\sum_{i=1}^{n} l\left(\gamma \mid\left[t_{i-1}, t_{i}\right]\right)$.

Let $\gamma:[a, b] \rightarrow X$ be rectifiable. As in [65] we show that there exists a unique path $\gamma^{0}:[0, c] \rightarrow X$ such that $\gamma=\gamma^{0} \circ h$, where $h:[a, b] \rightarrow[0, c]$ is increasing, $l\left(\gamma^{0} \mid[0, t]\right)=t$ for every $t \in[0, c]$ and we prove that $h=s_{\gamma}$ and $c=l(\gamma)$. Here $s_{\gamma}:[a, b] \rightarrow[0, l(\gamma)]$ is given by $s_{\gamma}(t)=l(\gamma \mid[a, t])$ for every $t \in[a, b]$ and is called the length function of $\gamma$. Then $s_{\gamma}$ is increasing and continuous and $d(\gamma(t), \gamma(s)) \leq s_{\gamma}(t)-s_{\gamma}(s)$ for every $a \leq s \leq t \leq b$ and if $h:\left[a^{\prime}, b^{\prime}\right] \rightarrow[a, b]$ is increasing (decreasing), then $l(\gamma \circ h)=l(\gamma)$. The path $\gamma^{0}:[0, l(\gamma)] \rightarrow X$ is called the normal representation of $\gamma$.

Let $\gamma:[a, b] \rightarrow X$ be rectifiable and $\rho: X \rightarrow[0, \infty]$ a Borel function. We set $\int_{\gamma} \rho d s=\int_{0}^{l(\gamma)} \rho\left(\gamma^{0}(t)\right) d t$ the line integral of $\rho$ over $\gamma$. If $\gamma:[a, b] \rightarrow X$ is locally rectifiable, we set $\int_{\gamma} \rho d s=\sup _{\beta} \int_{\beta} \rho d s$, where the supremum is taken over all closed subpaths $\beta$ of $\gamma$.

Let $D \subset X$ be open. We set $A(D)$ the set of all nonconstant path families in $D$. If $\Gamma \in A(D)$, we set $F(\Gamma)=\left\{\rho: X \rightarrow[0, \infty]\right.$ Borel function $\mid \int_{\gamma} \rho d s \geq 1$ for every $\gamma \in \Gamma$ locally rectifiable $\}$. If $\Gamma_{1}, \Gamma_{2} \in A(D)$, we say that $\Gamma_{1}>\Gamma_{2}$ if every path $\gamma_{1} \in \Gamma_{1}$ has a subpath $\gamma_{2} \in \Gamma_{2}$.

Let $p>1$ and $\omega: D \rightarrow[0, \infty]$ be $\mu$ measurable and finite $\mu$ a.e. We define the $p$-modulus of weight $\omega$ by

$$
M_{\omega}^{p}(\Gamma)=\inf _{\rho \in F(\Gamma)} \int_{X} \omega(x) \rho^{p}(x) d \mu \quad \text { if } \Gamma \in A(D) .
$$

If $F(\Gamma)=\phi$, we set $M_{\omega}^{p}(\Gamma)=0$. If $\omega=1$, we put

$$
M_{p}(\Gamma)=\inf _{\rho \in F(\Gamma)} \int_{X} \rho^{p}(x) d \mu \quad \text { if } \Gamma \in A(D) .
$$

We see that if $\Gamma_{1}, \Gamma_{2} \in A(D), \Gamma_{1}>\Gamma_{2}$, then $M_{\omega}^{p}\left(\Gamma_{1}\right) \leq M_{\omega}^{p}\left(\Gamma_{2}\right)$ and if $\Gamma=\bigcup_{k=1}^{\infty} \Gamma_{k}$ with $\Gamma_{k} \in A(D)$ for every $k \in \mathbf{N}$, then $M_{\omega}^{p}(\Gamma) \leq \sum_{k=1}^{\infty} M_{\omega}^{p}\left(\Gamma_{k}\right)$.

Let $E \subset X, p>1$ and $\omega: X \rightarrow[0, \infty]$ be $\mu$ measurable and finite $\mu$ a.e. We say that $M_{\omega}^{p}(E)=0$ if $M_{\omega}^{p}(\Gamma)=0$, where $\Gamma=\{\gamma:[0,1) \rightarrow X$ path $\mid \gamma$ has at least a limit point in $E\}$ and if $\omega=1$, we say that $M_{p}(E)=0$. We say that $M_{\omega}^{p}(E)>0$ if it is false that $M_{\omega}^{p}(E)=0$. It is clear that if $E=\left\{x_{n}\right\}_{n \in \mathbf{N}}$ and $M_{\omega}^{p}\left(x_{n}\right)=0$ for every $n \in \mathbf{N}$, then $M_{\omega}^{p}(E)=0$.

Here, if $\gamma:[0,1) \rightarrow X$ is an open path and there exists $t_{n} \rightarrow 1$ such that $\gamma\left(t_{n}\right) \rightarrow$ $x$, we say that $x$ is a limit point of $\gamma$. 
Example 1.1. Let $p<m, E \subset X$ such that $M_{m}(E)=0, D \subset X$ open such that $\int_{D \backslash E} \omega(x)^{\frac{m}{m-p}} d \mu<\infty$. Let $\Gamma=\{\gamma:[0,1) \rightarrow D \backslash E$ path $\mid \gamma(0) \in D \backslash E$ and $\gamma$ has at least a limit point in $E$ \} and let $\rho \in F(\Gamma)$. Then using Hölder's inequality, we have

$$
M_{\omega}^{p}(\Gamma) \leq \int_{D \backslash E} \omega(x) \rho^{p}(x) d \mu \leq\left(\int_{D \backslash E} \omega(x)^{\frac{m}{m-p}} d \mu\right)^{\frac{m-p}{m}}\left(\int_{D \backslash E} \rho(x)^{m} d \mu\right)^{\frac{p}{m}} .
$$

Since $M_{m}(\Gamma)=M_{m}(E)=0$ and $\rho \in F(\Gamma)$ was arbitrarily chosen, we see that $M_{\omega}^{p}(E)=0$.

Let $E, F \subset X$ and $U \subset X$ such that $E \cup F \subset \bar{U}$. We set $\Delta(E, F, U)=\{\gamma:[0,1] \rightarrow$ $\bar{U}$ path $\mid \gamma(0) \in E, \gamma(1) \in F$ and $\gamma((0,1)) \subset U\}$. As in [25], we set for $p>1$ and $E, F$ closed subsets of an open set $U \subset X$

$$
\operatorname{cap}_{p}(E, F, U)=\inf \int_{U} \rho^{p}(x) d \mu
$$

where the infimum is taken over all upper gradients of all functions $u: U \rightarrow \mathbf{R}$ such that $U \mid E \geq 1$ and $U \mid F \leq 1$. We see from Proposition 2.17 in [25] that if $X$ is proper and $\varphi$-convex, then $\operatorname{cap}_{p}(E, F, U)=M_{p}(\Delta(E, F, U))$.

Let $E \subset X$ closed, $E \subset A$ open and $\Gamma_{E}=\Delta(E, \complement A, A)$. We say that $\operatorname{cap}_{p}(E)=0$ if $\operatorname{cap}_{p}(E, \complement A, A)=0$ for every open set $E \subset A$ and if $X$ is proper and $\varphi$-convex, then $\operatorname{cap}_{p}(E, \complement A, A)=M_{p}\left(\Gamma_{E}\right)$ and hence a closed set $E \subset X$ is $\operatorname{such}_{\text {that }} \operatorname{cap}_{p}(E)=0$ if and only if $M_{p}(E)=0$. It is important in our paper to find conditions for a set $E \subset X$ to be such that $M_{\omega}^{p}(E)=0$, at least for a punctual set $E$.

Let $x \in X$ and $0<a<b$. We set $\Gamma_{x, a, b}=\Delta(\bar{B}(x, a), S(x, b), B(x, b))$. We set $L_{x, a, b}=\{\rho: X \rightarrow[0, \infty] \mid$ there exists a Borel function $\eta:(a, b) \rightarrow(0, \infty]$ such that $\int_{a}^{b} \eta(t) d t \geq 1$ and $\rho(z)=\eta(d(x, z))$ if $z \in C(x, a, b), \rho(z)=0$ otherwise $\}$. Here $C(x, a, b)=B(x, b) \backslash \bar{B}(x, a)$. We set if $\omega: D \rightarrow[0, \infty]$ is $\mu$ measurable and finite $\mu$ a.e.

$$
\Delta_{\omega}^{p}\left(\Gamma_{x, a, b}\right)=\inf _{\rho \in L_{x, a, b}} \int_{X} \omega(z) \rho^{p}(z) d \mu .
$$

We shall prove in Chapter 2 that $L_{x, a, b} \subset F\left(\Gamma_{x, a, b}\right)$ and hence

$$
M_{\omega}^{p}\left(\Gamma_{x, a, b}\right) \leq \Delta_{\omega}^{p}\left(\Gamma_{x, a, b}\right)
$$

We say that $M_{\omega}^{p}(x)=0$ if there exists $0<b_{0}<d(x, \partial D)$ such that $\lim _{a \rightarrow 0} M_{\omega}^{p}\left(\Gamma_{x, a, b}\right)=$ 0 for every fixed $0<b<b_{0}$ and we say that $\Delta_{\omega}^{p}(x)=0$ if there exists $0<b_{0}<$ $d(x, \partial D)$ such that $\lim _{a \rightarrow 0} \Delta_{\omega}^{p}\left(\Gamma_{x, a, b}\right)=0$ for every fixed $0<b<b_{0}$. We see from (1.2) that if $\Delta_{\omega}^{p}(x)=0$, then $M_{\omega}^{p}(x)=0$.

Let $D \subset X$ be a domain. We say that a $\mu$ measurable function $\omega: D \rightarrow[0, \infty]$ has finite mean oscillation at a point $x \in D$ (abbr. $\omega \in F M O(x)$ ) if there exists $\epsilon_{0}>0$ such that $\int_{B\left(x, \epsilon_{0}\right)} \omega(z) d \mu<\infty$ and

$$
\limsup _{\epsilon \rightarrow 0} f_{B(x, \epsilon)}\left|\omega(z)-\omega_{B(x, \epsilon)}\right| d \mu<\infty .
$$

Here, if $f: X \rightarrow \mathbf{R}$ is $\mu$ measurable, we set $f_{B}=\frac{1}{\mu(B)} \int_{B} f(z) d \mu$ for every ball $B \subset X$.

Using a result from Chapter 13 in [41], we have

Lemma 1.1. Let $X$ be an Ahlfors $Q$-regular metric measure space with $Q \geq 2$, $D \subset X$ a domain, $x \in D$ and $\omega \in F M O(x)$. Then $\Delta_{\omega}^{Q}(x)=0$. 
We found in Lemma 1.1 and Lemma 2.6 some conditions in order that $\Delta_{\omega}^{p}(x)=0$. We remark that if $\eta:(0, \infty) \rightarrow[0, \infty]$ is a Borel function such that $\int_{a}^{b} \eta(t) d t>0$ for every $0<a<b$ and $\int_{0}^{b} \eta(t) d t=\infty$, then

$$
\Delta_{\omega}^{p}\left(\Gamma_{x, a, b}\right) \leq \int_{C(x, a, b)} \omega(z) \eta(d(x, z))^{p} d \mu /\left(\int_{a}^{b} \eta(t) d t\right)^{p} .
$$

It results that if $\int_{B(x, b)} \omega(z) \eta(d(x, z))^{p} d \mu<\infty$ for some $b>0$, then $\Delta_{\omega}^{p}(x)=0$.

Let $D \subset X$ be open and $f: D \rightarrow Y$. We say that $f$ is open if $f$ carries open sets into open sets, we say that $f$ is discrete if either $f^{-1}(y)=\phi$, or $f^{-1}(y)$ is a discrete set in $D$ for every $y \in Y$ and we say that $f$ is a light map if $\operatorname{dim} f^{-1}(y) \leq 0$ for every $y \in Y$. Here, if $A \subset X, \operatorname{dim} A$ is the topological dimension of $A$ (see the monograph [28] for more information on dimension theory).

A metric measure space $X$ is Ahlfors $Q$-regular if there exists a Borel regular measure $\mu$ on $X$ and a constant $C_{0}$ such that

$$
\frac{1}{C_{0}} r^{Q} \leq \mu(B(x, r)) \leq C_{0} r^{Q} \text { for every ball } B(x, r) \subset X .
$$

Let $u: X \rightarrow \mathbf{R}$. A non-negative Borel measurable function $\rho: X \rightarrow[0, \infty]$ is said to be an upper gradient of $u$ if for every $x, y \in X$ and every rectifiable path $\gamma:[a, b] \rightarrow X$ with $\gamma(a)=x, \gamma(b)=y$, the following inequality holds:

$$
|u(x)-u(y)| \leq \int_{\gamma} \rho d s .
$$

The space $X$ is said to support a $(1, q)$-Poincaré inequality $(q>1)$ if there exists constants $C \geq 1$ and $r \geq 1$ such that for every bounded continuous functions $u: X \rightarrow \mathbf{R}$, all balls $B \subset X$ and all upper gradients $\rho$ of $u$ the following inequality holds true

$$
f_{B}\left|u(x)-u_{B}\right| d \mu \leq C d(B)\left(f_{r B} \rho^{q}(x) d \mu\right)^{\frac{1}{q}} .
$$

Here, if $A \subset X$, we denote by $d(A)$ the diameter of the set $A$.

In this paper we study the geometric properties of continuous, open light mappings $f: D \rightarrow Y$ satisfying the following relation:

$$
M_{q}(f(\Gamma)) \leq \gamma\left(M_{\omega}^{p}(\Gamma)\right) \text { for every } \Gamma \in A(D) .
$$

Here $D \subset X$ is a domain, $Y$ is Ahlfors $Q$-regular and supports a $(1, q)$-Poincaré inequality with $Q-1<q \leq Q, p>1, \omega: D \rightarrow[0, \infty]$ is a $\mu$-measurable function which is $\mu$-finite a.e., $\gamma:(0, \infty) \rightarrow(0, \infty)$ is increasing and $\lim _{t \rightarrow 0} \gamma(t)=0$.

If $\gamma(t)=t$ for $t>0$ and $p=q$, relation (1.3) is a Poleckii type modular inequality for general metric measure spaces. In [21] this relation is proved for quasiregular mappings $f: X \rightarrow Y$ between Ahlfors $p$-regular spaces. In [30] such a relation is proved for open, discrete mappings between Riemannian manifolds $M_{n} \neq \mathbf{R}^{n}$ and in [60] such a relation is proved for factor spaces $B^{n} / G$, where $B^{n}$ is the unit ball in $\mathbf{R}^{n}$ and $G$ is a Möbius group. This shows that there exist open, discrete mappings satisfying a Poleckii type modular inequality on general metric measure spaces and hence the theory of the class mappings satisfying relation (1.3) is effective.

It is interesting that even if $n \geq 3$ and $D \subset \mathbf{R}^{n}$ is a domain, a result of Wilson [68] shows that there exists a continuous, open, light mapping $f: D \subset \mathbf{R}^{n} \rightarrow \mathbf{R}^{n}$ such that $D=B_{f}=\{x \in D \mid f$ is not a local homeomorphism at $x\}$ and hence such a mapping is not discrete. We prove the following eliminability result: 
Theorem 1.1. Let $p>1, D \subset X$ a domain, $E \subset D$ closed and nowhere disconnecting, $\omega: D \rightarrow[0, \infty]$ be $\mu$ measurable and finite $\mu$ a.e. such that $M_{\omega}^{p}(E)=0$, let $f: D \backslash E \rightarrow Y$ be continuous, open and light and $x \in E$. Suppose that there exists a continuum $M \subset Y$ with Card $M>1$ and $r_{x}>0$ such that $\bar{B}\left(x, r_{x}\right) \subset D$ and $f\left(B\left(x, r_{x}\right) \backslash E\right) \subset Y \backslash M$ and there exists $\gamma:(0, \infty) \rightarrow(0, \infty)$ increasing such that $f$ satisfies condition (1.3). Then there exists $\lim _{y \rightarrow x} f(y) \in \bar{Y}$.

Remark 1.1. If $E=\{x\}$, we can replace condition (1.3) from the preceding theorem with a weaker one

$$
M_{q}\left(f\left(\Gamma_{x, a, b}\right)\right) \leq \gamma\left(\Delta_{\omega}^{p}\left(\Gamma_{x, a, b}\right)\right) \text { for every } 0<a<b<d(x, \partial D) .
$$

We also replace condition " $M_{\omega}^{p}(x)=0$ " with the condition " $\Delta_{\omega}^{p}(x)=0$ ".

Mappings satisfying condition (1.4) are called in [41] ring mappings in the point $x$. Theorem 1.1 was proved in [30] and [58] for bounded, open discrete mappings satisfying condition (1.4) and such that $\Delta_{\omega}^{p}(x)=0$. If $D \subset X$ is open, $E \subset D, x \in E$ and $f: D \backslash E \rightarrow Y$ is a mapping, we say that $x$ is an essential singularity of $f$ if there exists no $\lim _{y \rightarrow x} f(y)=l \in \bar{Y}$.

Theorem 1.2. Let $p>1, D \subset X$ a domain, $E \subset D$ closed and nowhere disconnecting, $\omega: D \rightarrow[0, \infty]$ be $\mu$ measurable and finite $\mu$ a.e. such that $M_{\omega}^{p}(E)=0$, let $f: D \backslash E \rightarrow Y$ be continuous open and light and let $x \in E$ be an essential singularity of $f$. Suppose that there exists $\gamma:(0, \infty) \rightarrow(0, \infty)$ increasing with $\lim _{t \rightarrow 0} \gamma(t)=0$ and such that $f$ satisfies condition (1.3). Then $\operatorname{dim}(Y \backslash f(B(x, r) \backslash E))=0$ for every $r>0$.

Remark 1.2. If $E=\{x\}$ we may replace in the preceding theorem condition (1.3) by condition (1.4). We also replace condition " $M_{\omega}^{p}(x)=0$ " with condition $" \Delta_{\omega}^{p}(x)=0 "$.

Our theorem extends a result from [61]. Of course, if $\operatorname{dim} Y \geq 1$, our theorem shows, as in the classical case of holmorphic or quasiregular mappings, that $f(B(x, r) \backslash E)$ is densely in $Y$ for every $r>0$. We prove the following equicontinuity result:

Theorem 1.3. Let $p>1, D \subset X$ a domain, $x \in D, \omega: D \rightarrow[0, \infty] \mu$ measurable and finite $\mu$ a.e. such that $\Delta_{\omega}^{p}(x)=0$. Let $W$ be a family of continuous, open, light mappings $f: D \rightarrow Y \backslash M_{f}$, where $M_{f}$ is a continuum with Card $M_{f}>1$ for every $f \in W$ and there exists $\delta>0, y \in Y$ and $R_{0}>0$ such that $d\left(M_{f}\right) \geq \delta$ and $M_{f} \cap B\left(y, R_{0}\right) \neq \phi$ for every $f \in W$ and $\{f(x)\}_{f \in W} \subset B\left(y, R_{0}\right)$. Suppose that there exists $\gamma:(0, \infty) \rightarrow(0, \infty)$ increasing with $\lim _{t \rightarrow \infty} \gamma(t)=0$ such that condition (1.4) is satisfied for every mapping $f \in W$ in the point $x$. Then the family $W$ is equicontinuous at $x$.

Remark 1.3. The preceding theorem was proved in [30] and [58] for uniformly bounded families $W$ of open, discrete mappings $f: D \rightarrow B\left(y, R_{0}\right) \backslash M_{f}$, for every $f \in W$, where $M_{f}$ is a continuum in $B\left(y, R_{0}\right)$ depending on $f$.

We prove a Picard type theorem.

Theorem 1.4. Let $X$ be such that $\bar{X} \neq X, E \subset X$, let $p>1$ and $\omega: X \rightarrow[0, \infty]$ be $\mu$ measurable and finite $\mu$ a.e. such that $M_{\omega}^{p}(E)=0$ and there exists $x \in X$ and $r>0$ such that $\lim _{R \rightarrow \infty} \Delta_{\omega}^{p}\left(\Gamma_{x, r, R}\right)=0$. Let $f: X \backslash E \rightarrow Y$ be continuous, open and light such that there exists $\gamma:(0, \infty) \rightarrow(0, \infty)$ increasing with $\lim _{t \rightarrow 0} \gamma(t)=0$ and $f$ satisfies condition (1.3). Then $\operatorname{dim}(Y \backslash f(X \backslash E))=0$. 
Remark 1.4. If $X$ is Ahlfors $p$-regular and $\omega=1$, the conditions from the preceding theorem are satisfied. The result from Theorem 1.4 was proved in [10] for mappings with finite distortion.

Let $D \subset X$ be a domain, $x \in \partial D$ and $f: D \rightarrow Y$. We define the cluster set $C(f, x)=\left\{y \in Y \mid\right.$ there exists $x_{p} \in D, x_{p} \neq x, x_{p} \rightarrow x$ such that $\left.f\left(x_{p}\right) \rightarrow y\right\}$. Let $F: \partial D \rightarrow \mathcal{P}(Y)$ be given by $F(z)=C(f, z)$ for every $z \in \partial D$. If $K \subset \partial D$, we set $C(f, x, K)=\bigcap_{m=1}^{\infty} \overline{F\left(U_{m} \cap(K \backslash\{x\})\right)}$, where the closure is taken in $Y$ and $\left(U_{m}\right)_{m \in \mathbf{N}}$ is a fundamental system of neighbourhoods of $x$. Here $\mathcal{P}(Y)$ is the family of all subsets of $Y$. If $\gamma:[0,1) \rightarrow D$ is a path and $\lim _{t \rightarrow 1} \gamma(t)=x$ and $w \in Y$ is such that $\lim _{t \rightarrow 1} f(\gamma(t))=w$, we say that $w$ is an asymptotic value of $f$ in $x$ and we set $A(f, x)=\{w \in Y \mid w$ is an asymptotic value of $f$ at $x\}$.

We say that the space $Y$ has property $\left(P_{q}\right), q>1$, if for every $y \in Y$ there exists $r_{y}>0$ and a constant $C$ depending on $y$ such that $M_{q}\left(\Delta\left(C_{1}, C_{2}, B(y, C r)\right)>0\right.$ for every non-degenerate disjoint continua $C_{1}, C_{2} \subset B(y, r)$ and every $0<r<r_{y}$. It is known that $\mathbf{R}^{n}$ has property $\left(P_{n}\right)$. We also see from page 17 in [22] that if $Y$ is of locally $q$-bounded geometry, then $Y$ has property $\left(P_{q}\right)$. As a particular case, let us point out that every Riemannian $q$ manifold has property $\left(P_{q}\right)$.

An important chapter in the theory of complex functions is dedicated to the study of cluster sets (see the book [42]). A classical theorem in this field is due to Nashiro. He proved in [42], page 14 that if $D \subset \mathbf{C}$ is a domain, $E \subset \partial D$ is compact and $\operatorname{cap}_{2}(E)=0, f: D \rightarrow \overline{\mathbf{C}}$ is meromorphic and $\gamma \in C(f, x) \backslash C(f, x, \partial D \backslash E)$ is an exceptional value of $f$ (i.e. $\alpha \notin \bigcap_{r>0} f(B(x, r) \cap D)$ ), then either $\alpha \in A(f, x)$, or there exists $x_{k} \in E, x_{k} \rightarrow x$ such that $x \in A\left(f, x_{k}\right)$ for every $k \in \mathbf{N}$. Some extensions of this result were given by Martio and Rickman in [38] for quasiregular mappings and in [10] by Cristea for mappings of finite distortion.

Theorem 1.5. Let $Y$ be a metric measure space having property $\left(P_{q}\right), q>1$, such that $B(y, r)$ is pathwise connected and such that $\operatorname{dim} S(y, r) \geq 1$ for every $y \in Y$ and every $r>0, p>1, D \subset X$ a domain such that $\operatorname{dim} \partial D \geq 1$, let $\omega: D \rightarrow[0, \infty]$ be $\mu$ measurable and finite $\mu$ a.e., $E \subset \partial D$ such that $\operatorname{dim} E=0$ and $M_{\omega}^{p}(E)=0$. Let $f: D \rightarrow Y$ be continuous, open light and suppose that there exists $\gamma:(0, \infty) \rightarrow(0, \infty)$ increasing with $\lim _{t \rightarrow 0} \gamma(t)=0$ such that $f$ satisfies condition (1.3).

Let $x \in(\partial D \backslash E)^{\prime}$ and $z \in C(f, x) \backslash\left(C(f, x, \partial D \backslash E) \cup \bigcap_{r>0} f(B(x, r) \cap D)\right)$. Then either $x \in E$ and $z \in A(f, x)$, or there exists $x_{k} \in E, x_{k} \rightarrow x$ such that $z \in A\left(f, x_{k}\right)$ for every $k \in \mathbf{N}$.

The next result extends a theorem which for plane meromorphic functions is known as Iversen's theorem and Cartwright's theorem. Our result also extends a theorem of Martio and Rickman from [38] established for quasiregular mappings and a theorem of Cristea from [10] established for mappings of finite distortion.

Theorem 1.6. Let $Y$ having property $\left(P_{q}\right), Q-1<q \leq Q$, such that $B(y, r)$ is pathwise connected and such that $\operatorname{dim} S(y, r) \geq 1$ for every $y \in Y$ and every $r>0, p>1, D \subset X$ a domain, $E$ is $D$ closed in $D$ and nowhere disconnecting, $\omega: D \rightarrow[0, \infty]$ be $\mu$ measurable and finite $\mu$ a.e. such that $M_{\omega}^{p}(E)=0$. Let $f: D \backslash E \rightarrow Y$ be continuous, open and light and $x$ an essential singularity of $f$. Suppose that there exists $\gamma:(0, \infty) \rightarrow(0, \infty)$ increasing with $\lim _{t \rightarrow 0} \gamma(t)=0$ and such that $f$ satisfies condition (1.3). Then, if $x$ is an isolated point of $E$, it results 
that $Y \backslash \bigcap_{r>0} f(B(x, r) \backslash E) \subset A(f, x)$ and in the general case there exists $x_{k} \in E$, $x_{k} \neq x, x_{k} \rightarrow x$ such that $Y \backslash \bigcap_{r>0} f(B(x, r) \backslash E) \subset A\left(f, x_{k}\right)$ for every $k \in \mathbf{N}$.

Remark 1.5. If in the preceding theorem $x$ is an isolated singularity of $f$ we may replace condition (1.3) by condition (1.4). We also replace condition " $M_{\omega}^{p}(x)=0$ " by condition " $\Delta_{\omega}^{p}(x)=0$ ". Some analog of Theorem 1.6 was proved in Theorem 1 from [57]. We also see that our Theorem 1.5 extends the classical result of Noshiro from [42], page 14 even in the Euclidean setting, since it works for "singular" sets $E \subset \partial D$ which might not always be compact.

The next theorem extends a result of Martio and Rickman from [38] concerning the density of the points $x \in S^{n}$ at which a quasiregular mapping $f: B^{n} \rightarrow B^{n}$ with $\operatorname{cap}\left(\complement f\left(B^{n}\right)\right)>0$ has some asymptotic values and a result of Cristea from [10] established for mappings of finite distortion.

Theorem 1.7. Let $D \subset X$ a domain, $B=\{b \in \partial D \mid$ there exists $\alpha:[0,1) \rightarrow D$ a path such that $\left.\lim _{t \rightarrow 1} \alpha(t)=b\right\}$, let $f: D \rightarrow Y$ be continuous, open and light and $E=\left\{b \in B \mid\right.$ there exists a path $\alpha:[0,1) \rightarrow D$ such that $\lim _{t \rightarrow 1} \alpha(t)=b$ and $\left.\lim _{t \rightarrow 1} f(\alpha(t))=l \in \bar{Y}\right\}$. Let $p \geq 2, \omega \in L_{\text {loc }}^{1}(D)$ such that $\omega(x)>0$ for $\mu$ a.e. $x \in D$, $M_{\omega}^{p}(b)=0$ for every $b \in B \backslash E, M_{\omega}^{p}(B \cap B(b, \epsilon))>0$ for every $b \in B$ and every $\epsilon>0$ and for every $b \in B \backslash E$ and every $\epsilon>0$, there exists a continuum $M$ depending on $b$ and $\epsilon$ such that Card $M>1$ and $f(D \cap B(b, \epsilon)) \subset Y \backslash M$. Suppose that there exists $\gamma:(0, \infty) \rightarrow(0, \infty)$ increasing with $\lim _{t \rightarrow 0} \gamma(t)=0$ such that $f$ satisfies condition (1.3). Then $M_{\omega}^{p}(B(b, \epsilon) \cap E)>0$ for every $b \in B$ and every $\epsilon>0$ and hence $E$ is dense in $B$.

\section{Preliminaries}

We first prove that $L_{x, a, b} \subset F\left(\Gamma_{x, a, b}\right)$ and hence we prove relation (1.2). We use the arguments from [65]. We say that $\gamma:[a, b] \rightarrow X$ is absolutely continuous if for every $\epsilon>0$ there exists $\delta_{\epsilon}>0$ such that $\sum_{i=1}^{n} d\left(\gamma\left(b_{i}\right), \gamma\left(a_{i}\right)\right)<\epsilon$ whenever $\Delta_{i}=\left[a_{i}, b_{i}\right]$ are non-overlaping subintervals of $[a, b]$ such that $\sum_{i=1}^{n}\left(b_{i}-a_{i}\right)<\delta_{\epsilon}$. As in [65] we see that such a path is rectifiable and if $\gamma:[a, b] \rightarrow X$ is a rectifiable path, then $s_{\gamma}$ is absolutely continuous if and only if $\gamma$ is absolutely continuous.

Let $f: X \rightarrow Y$ be a continuous mapping and let $x \in X$. We set

$$
L(x, f)=\limsup _{\substack{y \rightarrow x \\ z \rightarrow x \\ y \neq z}} \frac{d(f(y), f(z))}{d(y, z)} .
$$

We see that the mapping $x \rightarrow L(x, f)$ is a Borel function. Let $\gamma:[a, b] \rightarrow X$ be a path. We set $L_{\gamma}(t)=\lim \sup \frac{d(\gamma(u), \gamma(v))}{|v-u|}$ when $u \rightarrow t, u \geq t, v \rightarrow t, v \leq t, v \neq u$. As in [65], we have

Lemma 2.1. Let $\gamma:[a, b] \rightarrow X$ be a rectifiable path. Then $s_{\gamma}^{\prime}(t)=L_{\gamma}(t)$ a.e. in $[a, b]$.

Proof. Since $d(\gamma(t), \gamma(s)) \leq s_{\gamma}(t)-s_{\gamma}(s)$ for every $a \leq s \leq t \leq b$, we see that $L_{\gamma}(t) \leq s_{\gamma}^{\prime}(t)$ a.e. Let $A=\left\{t \in[a, b] \mid s_{\gamma}^{\prime}(t)\right.$ exists $\}$ and let $A_{k}=\left\{t \in A \mid \frac{s_{\gamma}(q)-s_{\gamma}(p)}{|p-q|} \geq\right.$ $\frac{d(\gamma(p), \gamma(q))}{|q-p|}+\frac{1}{k}$ whenever $a \leq p \leq t \leq q \leq b$ and $\left.0<|q-p|<\frac{1}{k}\right\}$ for every $k \in \mathbf{N}$. Let us fix $k \in \mathbf{N}$ and $\epsilon>0$.

Let $\Delta=\left(a=t_{0}<t_{1}<, \ldots,<t_{n}=b\right) \in \mathcal{D}([a, b])$ be such that $l(\gamma) \leq$ $\sum_{i=1}^{n} d\left(\gamma\left(t_{i-1}\right), \gamma\left(t_{i}\right)\right)+\frac{\epsilon}{k}$ if $0 \leq t_{i}-t_{i-1}<\frac{1}{k}$ for $i=1, \ldots, n$. Let $\Delta_{i}=\left[t_{i-1}, t_{i}\right]$ 
for $i=1, \ldots, n$. If $\Delta_{i} \cap A_{k} \neq \phi$, then $s_{\gamma}\left(t_{i}\right)-s_{\gamma}\left(t_{i-1}\right) \geq d\left(\gamma\left(t_{i-1}\right), \gamma\left(t_{i}\right)\right)+\frac{m_{1}\left(\Delta_{i}\right)}{k}$ and hence $m_{1}\left(A_{k}\right) \leq \sum_{\Delta_{i} \cap A_{k} \neq \phi} m_{1}\left(\Delta_{i}\right) \leq k\left(\sum_{i=1}^{n}\left(s_{\gamma}\left(t_{i}\right)-s_{\gamma}\left(t_{i-1}\right)-d\left(\gamma\left(t_{i-1}\right), \gamma\left(t_{i}\right)\right)\right) \leq\right.$ $\left.k\left(l(\gamma)-\sum_{i=1}^{n} d\left(\gamma\left(t_{i-1}\right), \gamma\left(t_{i}\right)\right)\right)\right) \leq \epsilon$. Letting $\epsilon \rightarrow 0$, we find that $m_{1}\left(A_{k}\right)=0$ for every $k \in \mathbf{N}$. Let $t \in A \backslash \bigcup_{k=1}^{\infty} A_{k}$. Then $s_{\gamma}^{\prime}(t) \leq L_{\gamma}(t)$ and the theorem is proved.

Lemma 2.2. Let $\gamma:[a, b] \rightarrow X$ be absolutely continuous and let $\rho: X \rightarrow[0, \infty]$ be a Borel function. Then $\int_{\gamma} \rho d s=\int_{a}^{b} \rho(\gamma(t)) L_{\gamma}(t) d t$.

Proof. Applying the change of variable formulae for real integrals we have

$$
\int_{\gamma} \rho d s=\int_{0}^{l(\gamma)} \rho\left(\gamma^{0}(t)\right) d t=\int_{a}^{b} \rho\left(\gamma^{0}\left(s_{\gamma}(t)\right) s_{\gamma}^{\prime}(t) d t=\int_{a}^{b} \rho(\gamma(t)) L_{\gamma}(t) d t .\right.
$$

Let $U \subset X$ be open, $\gamma:[a, b] \rightarrow U$ be rectifiable and let $f: U \rightarrow Y$ be continuous. We say that $f$ is absolutely continuous on $\gamma$ if $f \circ \gamma^{0}:[0, l(\gamma)] \rightarrow Y$ is absolutely continuous. Suppose that $f: U \rightarrow Y$ is $L$-lipschitz. Then

$$
d\left(f \circ \gamma^{0}(t), f \circ \gamma^{0}(s)\right) \leq L d\left(\gamma^{0}(t), \gamma^{0}(s)\right) \leq L l\left(\gamma^{0} \mid[s, t]\right) \leq L|t-s|
$$

for every $0 \leq s \leq t \leq l(\gamma)$ and hence if $f: U \rightarrow Y$ is $L$-lipschitz, then $f$ is absolutely continuous on $\gamma$.

As in [65], we have

Lemma 2.3. Let $U \subset X$ be open, $f: U \rightarrow X$ continuous, $\gamma:[a, b] \rightarrow U$ be a locally rectifiable path such that $f$ is absolutely continuous on every closed subpath of $\gamma$ and let $\rho: X \rightarrow[0, \infty]$ be a Borel function. Then $f \circ \gamma$ is locally rectifiable and $\int_{f \circ \gamma} \rho d s \leq \int_{\gamma} \rho(f(x)) L(x, f) d s$.

Proof. We can suppose that $\gamma$ is rectifiable. Since $f \circ \gamma^{0}$ is absolutely continuous, it is rectifiable and hence $f \circ \gamma=f \circ \gamma^{0} \circ s_{\gamma}$ is rectifiable and let $p=l(\gamma)$ and $q=l(f \circ \gamma)=l\left(f \circ \gamma^{0}\right)$. Let $s:[0, p] \rightarrow[0, q]$ be the length function of $f \circ \gamma^{0}$. Then $s$ is absolutely continuous and we see from Lemma 2.1 that $s^{\prime}(t)=L_{f \circ \gamma^{0}}(t)$ a.e. in $[0, p]$. Let $\beta=(f \circ \gamma)^{0}$. We have $f \circ \gamma=f \circ \gamma^{0} \circ s_{\gamma}=\left(f \circ \gamma^{0}\right)^{0} \circ s_{f \circ \gamma^{0}} \circ s_{\gamma}$ and also $f \circ \gamma=(f \circ \gamma)^{0} \circ s_{f \circ \gamma}$. Using the unicity of the normal representation of a path, we see that $(f \circ \gamma)^{0}=\left(f \circ \gamma^{0}\right)^{0}$. Then $\beta \circ s=(f \circ \gamma)^{0} \circ s=\left(f \circ \gamma^{0}\right)^{0} \circ s=f \circ \gamma^{0}$. Using the change of variable formulae for real integrals, we have

$$
\int_{f \circ \gamma} \rho d s=\int_{0}^{q} \rho(\beta(t)) d t=\int_{0}^{p} \rho\left(\beta(s(t)) s^{\prime}(t) d t=\int_{0}^{p} \rho\left(f \circ \gamma^{0}(t)\right) L_{f \circ \gamma^{0}}(t) d t .\right.
$$

Let $t \in[0, p]$ and let $r_{j} \searrow 0, s_{j} \nearrow 0$ such that $r_{j}-s_{j} \neq 0$ for every $j \in \mathbf{N}$. Since $\gamma^{0}$ is a normal representation, we see that $\gamma^{0}\left(t+r_{j}\right) \neq \gamma^{0}\left(t+s_{j}\right)$ for every $j \in \mathbf{N}$. We have

$$
\begin{aligned}
& \frac{d\left(f \circ \gamma^{0}\left(t+r_{j}\right), f \circ \gamma^{0}\left(t+s_{j}\right)\right)}{r_{j}-s_{j}} \\
& =\frac{d\left(f \circ \gamma^{0}\left(t+r_{j}\right), f \circ \gamma^{0}\left(t+s_{j}\right)\right)}{d\left(\gamma^{0}\left(t+r_{j}\right), \gamma^{0}\left(t+s_{j}\right)\right)} \frac{d\left(\gamma^{0}\left(t+r_{j}\right), \gamma^{0}\left(t+s_{j}\right)\right)}{r_{j}-s_{j}} \\
& \leq L\left(\gamma^{0}(t), f\right) L_{\gamma^{0}}(t) \leq L\left(\gamma^{0}(t), f\right)
\end{aligned}
$$

for every $j \in \mathbf{N}$ and hence $L_{f \circ \gamma^{0}}(t) \leq L\left(\gamma^{0}(t), f\right)$ for every $t \in[0, p]$. We proved that

$$
\int_{f \circ \gamma} \rho d s \leq \int_{0}^{p} \rho\left(f \circ \gamma^{0}(t)\right) L\left(\gamma^{0}(t), f\right) d t=\int_{\gamma} \rho(f(x)) L(x, f) d s .
$$


Lemma 2.4. Let $\gamma:[a, b] \rightarrow X$ be rectifiable, $x \in X$ such that $\operatorname{Im} \gamma \subset C(x, r, R)$ and let $\rho:[r, R] \rightarrow[0, \infty]$ be a Borel function. Then

$$
\int_{r}^{R} \rho(u) d u \leq\left|\int_{d(x, \gamma(a))}^{d(x, \gamma(b))} \rho(u) d u\right| \leq \int_{\gamma} \rho(d(x, z)) d s .
$$

Proof. Let $f: X \rightarrow[0, \infty], f(z)=d(x, z)$ for every $z \in X$. Then $f$ is 1 Lipschitz and $L(z, f) \leq 1$ for every $z \in X$ and $f$ is absolutely continuous on $\gamma$. Using Lemma 2.3, we have

$$
\int_{f \circ \gamma} \rho d s \leq \int_{\gamma} \rho(f(z)) L(z, f) d s \leq \int_{\gamma} \rho(d(x, z)) d s .
$$

Let $\beta=(f \circ \gamma)^{0}$ and $c=l(f \circ \gamma)$. Then $f \circ \gamma=(f \circ \gamma)^{0} \circ s_{f \circ \gamma},(f \circ \gamma)(a)=d(x, \gamma(a))$, $(f \circ \gamma)(b)=d(x, \gamma(b))$ and hence $\beta(0)=d(x, \gamma(a))$ and $\beta(c)=d(x, \gamma(b))$. We also see that $\beta$ is 1-Lipschiz and hence is absolutely continuous and $\left|\beta^{\prime}(t)\right| \leq 1$ a.e. Let $\rho_{k}=\min \{\rho, k\}$ for $k \in \mathbf{N}$. We may suppose that $d(x, \gamma(a)) \leq d(x, \gamma(b))$ and using the formulae from page 221 in [20], we have

$$
\begin{aligned}
\int_{d(x, \gamma(a))}^{d(x, \gamma(b))} \rho_{k}(t) d t & =\int_{\beta(0)}^{\beta(c)} \rho_{k}(t) d t=\int_{0}^{c} \rho_{k}(\beta(t)) \beta^{\prime}(t) d t \\
& \leq \int_{0}^{c} \rho(\beta(t)) d t=\int_{f \circ \gamma} \rho d s \leq \int_{\gamma}(\rho(d(x, z)) d s .
\end{aligned}
$$

Letting $k \rightarrow \infty$, we have

$$
\int_{r}^{R} \rho(u) d u \leq\left|\int_{d(x, \gamma(a))}^{d(x, \gamma(b))} \rho(u) d u\right| \leq \int_{\gamma} \rho(d(x, z)) d s .
$$

Remark 2.1. Let $x \in X$ and $0<a<b$. We see from Lemma 2.4 that $L_{x, a, b} \subset F\left(\Gamma_{x, a, b}\right)$ and hence relation (1.2) is proved.

The second main result of this chapter is the following:

Lemma 2.5. Let $D \subset X$ be open, $p \geq 2, \omega \in L_{\text {loc }}^{1}(D)$ such that $\omega(x)>0$ $\mu$ a.e. Then $M_{\omega}^{p}(x)=0$ if and only if there exists $0<b_{0}<d(x, \partial D)$ such that $\lim _{a \rightarrow 0} M_{\omega}^{p}\left(\Gamma_{x, a, b}\right)=0$ for every fixed $0<a<b<b_{0}<d(x, \partial D)$.

It results that $M_{\omega}^{p}(x)=0$ if $\Delta_{\omega}^{p}(x)=0$ and we can prove some conditions in order that $\Delta_{\omega}^{p}(x)=0$ and hence such that $M_{\omega}^{p}(x)=0$.

Lemma 2.6. Let $X$ be an Ahlfors $Q$-regular space, $D \subset X$ a domain, $x \in D$, $1<p<Q, \alpha>\frac{Q}{Q-p}$ and $\omega \in L^{\alpha}(D)$. Then $\Delta_{\omega}^{p}(x)=0$.

Proof. Let $d=d(x, \partial D)$ and $0<a<b<d$. Let $b_{k}=b 2^{-k}$ for $k \in \mathbf{N}$ and $\gamma=\frac{Q \alpha-p \alpha-Q}{\alpha-1}$. Letting $\eta(t)=\frac{1}{t}$ in the definition of $L_{x, a, b}$, we have

$$
\begin{aligned}
\Delta_{\omega}^{p}\left(\Gamma_{x, a, b}\right) & \leq \frac{1}{\left(\ln \frac{b}{a}\right)^{p}} \int_{C(x, a, b)} \omega(z) d(x, z)^{-p} d \mu \quad \text { (using Hölder's ineguality) } \\
& \leq \frac{\left(\int_{D} \omega(z)^{\alpha} d \mu\right)^{\frac{1}{\alpha}}}{\left(\ln \frac{b}{a}\right)^{p}}\left(\int_{C(x, a, b)} d(x, z)^{\frac{-p \alpha}{\alpha-1}} d \mu\right)^{\frac{\alpha-1}{\alpha}}
\end{aligned}
$$




$$
\begin{aligned}
& \leq \frac{\left(\int_{D} \omega(z)^{\alpha} d \mu\right)^{\frac{1}{\alpha}}}{\left(\ln \frac{b}{a}\right)^{p}}\left(\sum_{k=0}^{\infty} \int_{C\left(x, \frac{b_{k}}{2}, b_{k}\right)} d(x, z)^{\frac{-p \alpha}{\alpha-1}} d \mu\right)^{\frac{\alpha-1}{\alpha}} \\
& \leq \frac{\left(\int_{D} \omega(z)^{\alpha} d \mu\right)^{\frac{1}{\alpha}}}{\left(\ln \frac{b}{a}\right)^{p}}\left(\sum_{k=0}^{\infty} \mu\left(C\left(x, \frac{b_{k}}{2}, b_{k}\right)\right)\left(\frac{b_{k}}{2}\right)^{\frac{-p \alpha}{\alpha-1}}\right)^{\frac{\alpha-1}{\alpha}} \\
& \leq \frac{\left(\int_{D} \omega(z)^{\alpha} d \mu\right)^{\frac{1}{\alpha}}}{\left(\ln \frac{b}{a}\right)^{p}}\left(\sum_{k=0}^{\infty} C_{0} b_{k}^{Q} 2^{\frac{p \alpha}{\alpha-1}}\left(b_{k}\right)^{\frac{-p \alpha}{\alpha-1}}\right)^{\frac{\alpha-1}{\alpha}} \\
& \leq \frac{\left(\int_{D} \omega(z)^{\alpha} d \mu\right)^{\frac{1}{\alpha}}}{\left(\ln \frac{b}{a}\right)^{p}}\left(C_{0}\right)^{\frac{\alpha-1}{\alpha}} 2^{p} b^{\frac{Q \alpha-p \alpha-Q}{\alpha}}\left(\sum_{k=0}^{\infty} 2^{\frac{1}{2^{k \gamma}}}\right)^{\frac{\alpha-1}{\alpha}} \leq \frac{C}{\left(\ln \frac{b}{a}\right)^{p}} .
\end{aligned}
$$

The lemma is now proved.

Lemma 2.7. Let $\omega: X \rightarrow[0, \infty]$ be $\mu$ measurable and finite $\mu$ a.e., $x \in X$, $a>0, p>1,0 \leq \alpha<p-1$ and $M>0$ such that $\int_{B(x, \delta)} \omega(z) d \mu \leq M \delta^{p}(\ln \delta)^{\alpha}$ for every $0<a<\delta$. Then $\lim _{R \rightarrow \infty} \Delta_{\omega}^{p}\left(\Gamma_{x, a, R}\right)=0$.

Proof. Let $\eta:(a, \infty) \rightarrow(0, \infty), \eta(t)=\frac{1}{t \ln \left(\frac{t e}{a}\right)}$ for $t>a$. Then $\int_{a}^{R} \eta(t) d t=$ $\ln \ln \left(\frac{R e}{a}\right)$ and let $A_{k}=B\left(x, a e^{k+1}\right) \backslash \bar{B}\left(x, a e^{k}\right)$ for $k \in \mathbf{N}$. We see that $\frac{1}{d(x, z)} \leq \frac{e^{-k}}{a}$ and $\frac{1}{\ln \left(\frac{d(x, z)}{a} e\right)} \leq \frac{1}{k+1}$ if $z \in A_{k} k \in \mathbf{N}$. We have

$$
\begin{aligned}
\Delta_{\omega}^{p}\left(\Gamma_{x, a, R}\right) & \leq \int_{C(x, a, R)} \omega(z) \eta(d(x, z))^{p} d \mu /\left(\int_{a}^{R} \eta(t) d t\right)^{p} \\
& \leq \frac{1}{\left(\ln \ln \frac{R e}{a}\right)^{p}} \sum_{k=0}^{\infty} \int_{A_{k}} \frac{\omega(z)}{d(x, z)^{p} \ln \left(\frac{d(x, z)}{a} e\right)^{p}} d \mu \\
& \leq \frac{1}{\left(\ln \ln \frac{R e}{a}\right)^{p}} \sum_{k=0}^{\infty} \frac{1}{\left(a e^{k}\right)^{p}\left(k+1_{)}^{p}\right.} \int_{A_{k}} \omega(z) d \mu \\
& \leq \frac{1}{\left(\ln \ln \frac{R e}{a}\right)^{p}} \sum_{k=0}^{\infty} \frac{M\left(a e^{k+1}\right)^{p}\left(\ln \left(a e^{k+1}\right)\right)^{\alpha}}{\left(a e^{k}\right)^{p}(k+1)^{p}} \simeq \frac{C \sum_{k=1}^{\infty} \frac{1}{k^{p-\alpha}}}{\left(\ln \ln \left(\frac{R e}{a}\right)\right)^{p}}
\end{aligned}
$$

The theorem is now proved.

Let $f: X \rightarrow Y$ be continuous, open and light. A domain $D \subset X$ is called normal if $\bar{D}$ is compact and $\partial f(D)=f(\partial D)$. We see from page 186 in [67] (see also Lemma 2.7 in [37]) that if $D \subset X$ is a normal domain, $p:[0,1] \rightarrow f(D)$ is a path and $x \in D$ is such that $f(x)=p(0)$, then there exists a path $q:[0,1] \rightarrow D$ such that $q(0)=x$ and $f \circ q=p$.

Let $f: X \rightarrow Y$ be continuous, open and light and $p:[0,1] \rightarrow Y$ be a path and let $x \in X$ be such that $f(x)=p(0)$. We say that $q:[0, a) \rightarrow X$ is a maximal lifting of $p$ from $x$ if $0<a \leq 1, q(0)=x, f \circ q=p \mid[0, a)$ and $q$ is maximal with this property. As in Lemma 3.12 in [36] we show that if $p:[0,1] \rightarrow Y$ is a path, $x \in X$ and $f: X \rightarrow Y$ is continuous, open and light such that $f(x)=p(0)$, then we always find a maximal lifting of $p$ from $x$. We say that we cannot lift a path $p:[0,1] \rightarrow Y$ from a point $x \in X$ such that $f(x)=p(0)$ if we cannot find a path $q:[0,1] \rightarrow X$ such that $q(0)=x$ and $f \circ q=p$. 
A set $E \subset X$ is nowhere disconnecting if Int $E=\phi$ and for every domain $D \subset X$ it results that $D \backslash E$ is pathwise connected. If $X$ is a $n$-dimensional manifold, $E$ is closed and $\operatorname{dim} E \leq n-2$, then $E$ is nowhere disconnecting (see Theorem IV.4, page 48 in [28]). However, it is possible that $\operatorname{dim} X=m \geq 1, E$ is a punctual set and $E$ disconnects the space $X$. Indeed, let $X=B_{1} \cup B_{2} \cup\{x\}$, where $B_{1}, B_{2}$ are open balls in $\mathbf{R}^{n}$ such that $B_{1} \subset \complement \bar{B}_{2}, \bar{B}_{1} \cap \bar{B}_{2}=\{x\}$ and the topology and metric on $X$ is Euclidean.

If $A, B \subset X$, we set $d(A, B)$ the distance between $A$ and $B$. If $A \subset X$ and $r>0$, we set $B(A, r)=\{y \in X \mid$ there exists $x \in A$ such that $d(x, y)<r\}$. The inclusion $D \subset \subset X$ means that $D$ is open and $\bar{D}$ is a compact subset of $X$. If $D \subset X$ is open, $p>1$ and $\omega: D \rightarrow[0, \infty]$ is $\mu$ measurable and $0<\omega(x)<\infty$ for $\mu$ a.e. $x \in D$, we let $L_{\omega}^{p}(D)=\left\{f: D \rightarrow \mathbf{R}\left|\int_{D} \omega(x)\right| f^{p}(x) \mid d \mu<\infty\right\}$. Then $L_{\omega}^{p}(D)$ is a Banach space with the norm $\|f\|_{\omega}^{p}=\left(\int_{D} \omega(x)|f(x)|^{p} d \mu\right)^{\frac{1}{p}}$.

We shall use the fundamental theorem from [3].

Theorem A. Let $p>1$ and $X$ an Ahlfors $Q$-regular space that supports a $(1, p)$ Poincaré inequality with $Q-1<p \leq Q$, let $R>0$ and $E, F \subset B(x, R)$ be continua. Then there exists a constant $C_{1}>0$ such that

$$
C_{1} \min \{d(E), d(F)\} R^{Q-p-1} \leq M_{p}(\Delta(E, F, Y)) .
$$

Lemma 2.8. Let $M \subset X$ be a continua such that $B(x, R) \cap M \neq \phi, \complement \bar{B}(x, 3 R) \cap$ $M \neq \phi$. Then there exists a continuum $M_{0} \subset M \cap \overline{C(x, R, 3 R)}$ such that $d\left(M_{0}\right) \geq R$.

Proof. We see that $S(x, r) \cap M \neq \phi$ for every $R<r<3 R$ and let $M_{0}$ be a component of $M \cap \bar{C}(x, R, 3 R))$ which intersects $S(x, 2 R)$. We see from (10.1), page 16 in [67] that $M_{0} \cap \partial C(x, R, 3 R) \neq \phi$ and hence either $M_{0} \cap S(x, R) \neq \phi$, or $M_{0} \cap S(x, 3 R) \neq \phi$. It results that $d\left(M_{0}\right) \geq R$ and $M_{0} \subset \bar{C}(x, R, 3 R)$.

Lemma 2.9. Let $D \subset X$ be open, $\omega \in L_{\text {loc }}^{1}(D), p>1$ and let $\Gamma \in A(D)$. Then, for every $\epsilon>0$ and every $\rho \in F(\Gamma)$ there exists $\rho \leq \eta$ lower semicontinuous such that $\int_{X} \omega(x) \eta^{p}(x) d \mu \leq \int_{X} \omega(x) \rho^{p}(x) d \mu+\epsilon$.

Proof. Let $\rho \in F(\Gamma)$ such that $\rho^{p}=\sum_{i=1}^{\infty} c_{i} \chi_{E_{i}}$, where $c_{i}>0$ and $E_{i}$ are measurable such that $\bar{E}_{i}$ are compact for every $i \in \mathbf{N}$. Let $\epsilon>0$ and $E_{i} \subset V_{i}$ open sets such that $0 \leq \int_{V_{i}} \omega(x) d \mu-\int_{E_{i}} \omega(x) d \mu \leq \frac{\epsilon}{c_{i} 2^{l+1}}$ for every $i \in \mathbf{N}$. Let $\eta^{p}=\sum_{i=1}^{\infty} c_{i} \chi_{V_{i}}$. Then $\rho \leq \eta, \eta$ is lower semicontinuous and

$$
\begin{aligned}
0 & \leq \int_{X} \omega(x) \eta^{p}(x) d \mu-\int_{X} \omega(x) \rho^{p}(x) d \mu=\sum_{i=1}^{\infty} c_{i} \int_{V_{i}} \omega(x) d \mu-\sum_{i=1}^{\infty} c_{i} \int_{E_{i}} \omega(x) d \mu \\
& =\sum_{i=1}^{\infty} c_{i}\left(\int_{V_{i}} \omega(x) d \mu-\int_{E_{i}} \omega(x) d \mu\right) \leq \sum_{i=1}^{\infty} \frac{\epsilon}{2^{i+1}}=\epsilon
\end{aligned}
$$

Lemma 2.10. Let $D \subset \subset X$ be an open set, $\omega: D \rightarrow[0, \infty] \mu$ measurable and finite $\mu$ a.e., $p>1$ and let $\Gamma \in A(D)$ be such that $l(\gamma) \geq \delta>0$ for every $\gamma \in \Gamma$. Then $M_{\omega}^{p}(\Gamma) \leq \frac{1}{\delta^{p}} \int_{D} \omega(x) d \mu$.

Proof. Let $\rho: X \rightarrow[0, \infty], \rho(x)=\frac{1}{\delta}$ for $x \in D, \rho(x)=0$ otherwise. Then $\rho \in F(\Gamma)$ and $M_{\omega}^{p}(\Gamma) \leq \int_{X} \rho^{p}(x) \omega(x) d \mu \leq \frac{1}{\delta^{p}} \int_{D} \omega(x) d \mu$.

A Fuglede type theorem and a Ziemer type theorem hold as in the classical case. We give here a proof for the sake of completeness. 
Theorem 2.1. (Fuglede's theorem) Let $f_{k}: X \rightarrow \mathbf{R}$ be a sequence of Borel functions which converges to a Borel function $f: X \rightarrow \mathbf{R}$ in $L_{\omega}^{p}(X)$. Here $p>1$ and $\omega: X \rightarrow[0, \infty]$ is $\mu$ measurable and finite $\mu$ a.e. Then there exists a subsequence $\left(f_{k j}\right)_{j \in \mathbf{N}}$ of $\left(f_{k}\right)_{k \in \mathbf{N}}$ such that $\int_{\gamma}\left|f_{k_{j}}-f\right| d s \rightarrow 0$ for all locally rectifiable paths $\gamma$ in $X$ except for a family $\Gamma$ with $M_{\omega}^{p}(\Gamma)=0$.

Proof. Let $\left(f_{k_{j}}\right)_{j \in \mathbf{N}}$ be such that $\int_{X} \omega(x)\left|f_{k_{j}}(x)-f(x)\right|^{p} d \mu \leq 2^{-p_{j}-j}$ for all $j \in \mathbf{N}$. Let $\Gamma=\left\{\gamma:[0,1] \rightarrow X\right.$ locally rectifiable path $\left.\left|\int_{\gamma}\right| f_{k_{j}}-f \mid d s \nrightarrow 0\right\}$. Let $\Gamma_{j}=\left\{\gamma:[0,1] \rightarrow X\right.$ locally rectifibale path $\left.\left|\int_{\gamma}\right| f_{k_{j}}-f \mid d s \geq \frac{1}{2^{j}}\right\}$ for $j \in \mathbf{N}$. Then $2^{j}\left|f_{k_{j}}-f\right| \in F\left(\Gamma_{j}\right)$ for every $j \in \mathbf{N}$ and $M_{\omega}^{p}\left(\Gamma_{j}\right) \leq 2^{p j} \int_{X} \omega(x)\left|f_{k_{j}}-f\right|^{p}(x) d \mu \leq \frac{1}{2^{j}}$ for every $j \in \mathbf{N}$. We see that $\Gamma \subset \bigcup_{j=i}^{\infty} \Gamma_{j}$ for every $i \in \mathbf{N}$ and hence $M_{\omega}^{p}(\Gamma) \leq$ $\sum_{j=i}^{\infty} M_{\omega}^{p}\left(\Gamma_{j}\right) \leq \sum_{j=i}^{\infty} \frac{1}{2^{j}}=\frac{1}{2^{i-1}}$ for every $i \geq 1$. We proved that $M_{\omega}^{p}(\Gamma)=0$.

Theorem 2.2. (Ziemer's theorem) Let $p \geq 2, \omega: X \rightarrow[0, \infty] \mu$ measurable such that $0<\omega(x)<\infty$ for $\mu$ a.e. $x \in X$ and let $\Gamma_{m} \in A(X)$ be such that $\Gamma_{m} \subset \Gamma_{m+1}$ for every $m \in \mathbf{N}$ and $\Gamma=\bigcup_{m=1}^{\infty} \Gamma_{m}$. Then $M_{\omega}^{p}(\Gamma)=\lim _{m \rightarrow \infty} M_{\omega}^{p}\left(\Gamma_{m}\right)$.

Proof. We see that $M_{\omega}^{p}\left(\Gamma_{m}\right) \nearrow I \leq M_{\omega}^{p}(\Gamma)$. Let us show that $M_{\omega}^{p}(\Gamma) \leq I$ and we can suppose that $I<\infty$. Let $\rho_{m} \in F\left(\Gamma_{m}\right)$ be such that $\int_{X} \omega(x) \rho_{m}(x)^{p} d \mu \leq$ $M_{\omega}^{p}\left(\Gamma_{m}\right)+\frac{1}{2^{m}}$ for every $n \in \mathbf{N}$. Using Clarkson's inequality, we have

$$
\left(\left\|\frac{\rho_{i}+\rho_{j}}{2}\right\|_{\omega}^{p}\right)^{p}+\left(\left\|\frac{\rho_{i}-\rho_{j}}{2}\right\|_{\omega}^{p}\right)^{p} \leq \frac{1}{2}\left(\left(\left\|\rho_{i}\right\|_{\omega}^{p}\right)^{p}+\left(\left\|\rho_{j}\right\|_{\omega}^{p}\right)^{p}\right)
$$

If $i>j$, then $\frac{\rho_{i}+\rho_{j}}{2} \in F\left(\Gamma_{j}\right)$ and hence

$$
\begin{aligned}
& M_{\omega}^{p}\left(\Gamma_{j}\right)+\int_{X} \omega(x)\left\|\frac{\rho_{i}-\rho_{j}}{2}(x)\right\|^{p} d \mu \\
& \leq \int_{X} \omega(x)\left\|\frac{\rho_{i}+\rho_{j}}{2}(x)\right\|^{p} d \mu+\int_{X} \omega(x)\left\|\frac{\rho_{i}-\rho_{j}}{2}(x)\right\|^{p} d \mu \\
& \leq \frac{1}{2}\left(\int_{X} \omega(x) \rho_{i}(x)^{p} d \mu+\int_{X} \omega(x) \rho_{j}(x)^{p} d \mu\right) \\
& \leq \frac{1}{2}\left(M_{\omega}^{p}\left(\Gamma_{i}\right)+\frac{1}{2^{i}}+M_{\omega}^{p}\left(\Gamma_{j}\right)+\frac{1}{2^{j}}\right)
\end{aligned}
$$

for $i>j$. Since $M_{\omega}^{p}\left(\Gamma_{j}\right) \leq I<\infty$, we have

$$
\left(\left\|\rho_{i}-\rho_{j}\right\|_{\omega}^{p}\right)^{p} \leq \frac{1}{2}\left(M_{\omega}^{p}\left(\Gamma_{i}\right)-M_{\omega}^{p}\left(\Gamma_{j}\right)+\frac{1}{2^{i}}+\frac{1}{2^{j}}\right)
$$

for $i>j$. Since $M_{\omega}^{p}\left(\Gamma_{j}\right) \nearrow I<\infty$, we see that $\left(\rho_{i}\right)_{i \in \mathbf{N}}$ is a Cauchy sequence in the Banach space $L_{\omega}^{p}(X)$ and hence there exists $\rho \in L_{\omega}^{p}(X)$ such that $\rho_{i} \rightarrow \rho$ in $L_{\omega}^{p}(X)$. Using Fuglede's theorem we find a subsequence $\left(\rho_{i_{k}}\right)_{k \in \mathbf{N}}$ of $\left(\rho_{i}\right)_{i \in \mathbf{N}}$ such that if $\tilde{\Gamma}=\left\{\gamma \in \Gamma\left|\int_{\gamma}\right| \rho_{i_{k}}-\rho \mid d s \nrightarrow 0\right\}$, then $M_{\omega}^{p}(\tilde{\Gamma})=0$.

Let $\gamma \in \Gamma \backslash \tilde{\Gamma}$ and $\epsilon>0$. There exists $m_{\epsilon} \in \mathbf{N}$ such that $\int_{\gamma}\left|\rho_{i_{k}}-\rho\right| d s \leq \epsilon$ for $k \geq m_{\epsilon}$ and let $k_{\epsilon} \geq m_{\epsilon}$ be such that $\gamma \in \Gamma_{i_{k}}$ for $k \geq k_{\epsilon}$. Let $k \geq k_{\epsilon}$. Then $\int_{\gamma} \rho d s \geq \int_{\gamma} \rho_{i_{k}}-\int_{\gamma}\left|\rho_{i_{k}}-\rho\right| d s \geq 1-\epsilon$. Letting $\epsilon \rightarrow 0$, we find that $\int_{\gamma} \rho d s \geq 1$ for 
every $\gamma \in \Gamma \backslash \tilde{\Gamma}$ and hence $\rho \in F(\Gamma \backslash \tilde{\Gamma})$. Then

$$
\begin{aligned}
M_{\omega}^{p}(\Gamma) & \leq M_{\omega}^{p}(\Gamma \backslash \tilde{\Gamma})+M_{\omega}^{p}(\tilde{\Gamma})=M_{\omega}^{p}(\Gamma \backslash \tilde{\Gamma}) \leq \int_{X} \omega(x) \rho^{p}(x) d \mu \\
& =\left(\|\rho\|_{\omega}^{p}\right)^{p} \leq\left(\left\|\rho_{i}\right\|_{\omega}^{p}\right)+\left(\left\|\rho-\rho_{i}\right\|_{\omega}^{p}\right)^{p} \leq\left(M_{\omega}^{p}\left(\Gamma_{i}\right)+\frac{1}{2^{i}}\right)^{\frac{1}{p}}+\left(\left\|\rho_{i}-\rho\right\|_{\omega}^{p}\right)^{p} .
\end{aligned}
$$

Letting $i \rightarrow \infty$, we find that $M_{\omega}^{p}(\Gamma) \leq I$. The theorem is now proved.

Theorem 2.3. Let $X$ such that there exists $D_{k} \subset \subset X$ such that $D_{k} \nearrow X$, let $\omega \in L_{\text {loc }}^{1}(D), \omega>0$ a.e., let $p \geq 2$, let $\Gamma \in A(X)$ and let $\Gamma^{r}=\{\gamma \in \Gamma \mid$ $\gamma$ is rectifiable $\}$. Then $M_{\omega}^{p}(\Gamma)=M_{\omega}^{p}\left(\Gamma^{r}\right)$.

Proof. Let $\Gamma_{m}=\left\{\gamma \in \Gamma \mid \operatorname{Im} \gamma \subset D_{m}\right\}$ for $m \in \mathbf{N}$. Let $m \in \mathbf{N}$ be fixed, $\Gamma_{m}^{r}=\left\{\gamma \in \Gamma_{m} \mid \gamma\right.$ is rectifiable $\}$ and $\rho_{m} \in F\left(\Gamma_{m}^{r}\right)$. Let $\epsilon>0$ and $\eta_{m}: X \rightarrow[0, \infty]$, $\eta_{m}=\chi_{D_{m}}$ and $\rho_{\epsilon, m}=\left(\rho_{m}^{p}+\epsilon^{p} \eta_{m}^{p}\right)^{\frac{1}{p}}$. Let $\gamma \in \Gamma_{m}^{r}$. Then $1 \leq \int_{\gamma} \rho_{m} d s \leq \int_{\gamma} \rho_{\epsilon, m} d s$. If $\gamma \in \Gamma_{m} \backslash \Gamma_{m}^{r}$, then $1 \leq \infty=\epsilon \int_{\gamma} \eta_{m} d s \leq \int_{\gamma} \rho_{\epsilon, m} d s$ and this shows taht $\rho_{\epsilon, m} \in F\left(\Gamma_{m}\right)$. We find that

$$
M_{\omega}^{p}\left(\Gamma_{m}\right) \leq \int_{X} \omega(x) \rho_{\epsilon, m}(x)^{p} d \mu=\int_{X} \omega(x) \rho_{m}^{p}(x) d \mu+\epsilon^{p} \int_{D_{m}} \omega(x) d \mu .
$$

Letting $\epsilon \rightarrow 0$, we see that $M_{\omega}^{p}\left(\Gamma_{m}\right) \leq M_{\omega}^{p}\left(\Gamma_{m}^{r}\right)$ for every $m \in \mathbf{R}$. Letting $m \rightarrow \infty$ and using Ziemer's theorem, we obtain that $M_{\omega}^{p}(\Gamma) \leq M_{\omega}^{p}\left(\Gamma^{r}\right) \leq M_{\omega}^{p}(\Gamma)$ and hence $M_{\omega}^{p}(\Gamma)=M_{\omega}^{p}\left(\Gamma^{r}\right)$.

Theorem 2.4. Let $C_{0}, C_{1}$ be disjoint continua in $X r=d\left(C_{0}, C_{1}\right), D \subset \subset X$ such that $C_{0} \cup C_{1} \subset D$, let $p \geq 2, \omega \in L_{l o c}^{1}(D), \Gamma=\Delta\left(C_{0}, C_{1}, D\right)$ and let $\Gamma_{\delta}=$ $\Delta\left(B\left(C_{0}, \delta\right), B\left(C_{1}, \delta\right), D\right)$ for $0<\delta<\frac{r}{4}$. Then $\lim _{\delta \rightarrow 0} M_{\omega}^{p}\left(\Gamma_{\delta}\right)=M_{\omega}^{p}(\Gamma)$.

Proof. Using Theorem 2.3, we can suppose that every path $\gamma \in \Gamma$ is rectifiable. Let $D_{m}=\left\{x \in D \mid d\left(x, C_{0} \cup C_{1}\right)>\frac{1}{m}\right\}$ for every $m \in \mathbf{N}$. Then $D_{m}$ are open sets and $D_{m} \nearrow D$. Let $\Gamma_{m}=\{\beta$ path $\mid$ there exists $\gamma:[0,1] \rightarrow D$ rectifiable, $\gamma \in \Gamma$ and $0 \leq \alpha_{\gamma} \leq \beta_{\gamma} \leq 1$ such that $\beta=\gamma \mid\left[\alpha_{\gamma}, \beta_{\gamma}\right], \gamma\left(\alpha_{\gamma}\right) \in \partial D_{m}, \gamma\left(\beta_{\gamma}\right) \in \partial D_{m}$ and $\left.\gamma\left(\left(\alpha_{\gamma}, \beta_{\gamma}\right)\right) \subset D_{m}\right\}$ for $m \in \mathbf{N}$. Then $\Gamma>\Gamma_{m+1}>\Gamma_{m}$ for every $m \in \mathbf{N}$ and hence $M_{\omega}^{p}(\Gamma) \leq M_{\omega}^{p}\left(\Gamma_{m+1}\right) \leq M_{\omega}^{p}\left(\Gamma_{m}\right)$ for every $m \in \mathbf{N}$ and this shows that there exists $\lim _{m \rightarrow \infty} M_{\omega}^{p}\left(\Gamma_{m}\right) \geq M_{\omega}^{p}(\Gamma)$. Let $\epsilon>0$. Using Lemma 2.7, we find $\eta \in F(\Gamma)$ lower semicontinuous such that $\int_{X} \omega(x) \eta^{p}(x) d \mu \leq M_{\omega}^{p}(\Gamma)+\frac{\epsilon}{2}$.

Let $\lambda_{m}=\sup \left\{\lambda>0 \mid \int_{\gamma} \eta d s \geq \lambda\right.$ for every $\left.\gamma \in \Gamma_{m}\right\}$ for $m \in \mathbf{N}$. We see that $\lambda_{m+1} \geq \lambda_{m}$ for every $m \in \mathbf{N}$ and let $\lambda=\lim _{m \rightarrow \infty} \lambda_{m}$. We show that $\lambda \geq 1$. Indeed, suppose otherwise that $\lambda<1$ and let $0 \leq \lambda<\rho<1$. We can find paths $\gamma_{m} \in \Gamma_{m}$ such that $\int_{\gamma_{m}} \eta d s \leq \rho$ for every $m \in \mathbf{N}$ and we can suppose that $\gamma_{m}=\gamma_{m}^{\circ}$ for every $m \in \mathbf{N}$.

Let us fix $m \in \mathbf{N}$. We define $\gamma_{q m}:[0, \infty] \rightarrow D_{m}$ in the following way: Let $0 \leq \alpha_{q m} \leq \beta_{q m} \leq l\left(\gamma_{m}\right)$ be the greatest, respectively the least $t \in\left[0, l\left(\gamma_{m}\right)\right]$ such that $\gamma_{m}(t) \in \partial D_{q}$ for $q=1, \ldots, m$ and we set $\gamma_{q m}=\gamma_{m} \mid\left[\alpha_{q m}, \beta_{q m}\right]$ and $\gamma_{q m}$ is constant on $\left[0, \alpha_{q m}\right]$ and $\left[\beta_{q m}, \infty\right)$ and $\gamma_{q m}$ is continuous on $[0, \infty)$ for $q=1, \ldots, m$. We see that $\gamma_{k m}$ is a subpath of $\gamma_{p m}$ if $1 \leq k \leq p \leq m$ and $\gamma_{k m}$ is a subpath of $\gamma_{m}$ for $k=1, \ldots, m$ and $\gamma_{k, m} \in \Gamma_{k}$ for $k=1, \ldots, m$.

The family $\left(\gamma_{1 m}\right)_{m \in \mathbf{N}}$ is a 1-lipschitzian family and hence is equicontinuous. Using Ascoli's theorem, we obtain a sequence $\left(\gamma_{1 m}\right)_{m \in J_{1}}$ with $J_{1} \subset \mathbf{N}$ and a path $\beta^{1}:[0, \infty) \rightarrow \bar{D}$ such that $\gamma_{1 m} \rightarrow \beta^{1}$ if $m \in J_{1}, m \rightarrow \infty$. Taking a subsequence, we 
can presume that $\alpha_{1 m} \rightarrow a_{1}, \beta_{1 m} \rightarrow b_{1}$ for $m \in J_{1}, m \rightarrow \infty$ and that $\beta^{1}$ is constant outside $\left[a_{1}, b_{1}\right]$.

The family $\left(\gamma_{2 m}\right)_{m \in J_{1}}$ is equicontinuous and using Ascoli's theorem we find $J_{2} \subset$ $J_{1}$ an increasing sequence of natural numbers such that the family $\left(\gamma_{2 m}\right)_{m \in J_{2}}$ converges uniformly to a path $\beta^{2}:[0, \infty) \rightarrow \bar{D}$ and we can suppose that the first number from $J_{2}$ is the first number from $J_{1}$, that $\alpha_{2 m} \rightarrow a_{2}, \beta_{2 m} \rightarrow b_{2}$ if $m \in J_{2}, m \rightarrow \infty$, $a_{2} \leq a_{1} \leq b_{1} \leq b_{2}$ and that $\beta^{2}$ is constant outside $\left[a_{2}, b_{2}\right]$.

We continue the process of infinite. At step $k$ we find $J_{k} \subset J_{k-1} \subset, \ldots, \subset J_{1}$ sets of decreasing natural number and the first $k-1$ numbers from $J_{k}$ are the first $k-1$ number from $J_{k-1}$ and the family $\left(\gamma_{k m}\right)_{m \in J_{k}}$ converges uniformly to a path $\beta^{k}:[0, \infty) \rightarrow \bar{D}$. We can suppose that $\alpha_{k m} \rightarrow a_{k}, \beta_{k m} \rightarrow b_{k}$ for $m \in J_{k}, m \rightarrow \infty$, that $a_{k} \leq a_{k-1} \leq, \ldots, \leq a_{1} \leq b_{1} \leq, \ldots, \leq b_{k-1} \leq b_{k}$ and that $\beta^{k}$ is constant outside $\left[a_{k}, b_{k}\right]$.

Let $p_{k}$ be the $k$-th term from $J_{k}$ for $k \in \mathbf{N}$ and $J=\left\{p_{1}, p_{2}, \ldots, p_{k}, \ldots\right\}$. Then $J \subset J_{k}$ for every $k \in \mathbf{N}, \gamma_{k m} \rightarrow \beta^{k}$ uniformly on $\left[a_{k}, b_{k}\right], \beta^{k}\left|\left[a_{k}, b_{k}\right]=\beta^{k+1}\right|\left[a_{k}, b_{k}\right]$ for every $k \in \mathbf{N}$. We can correctly define $\beta:[0, \infty) \rightarrow \bar{D}$ by $\beta\left|\left[a_{k}, b_{k}\right]=\beta^{k}\right|\left[a_{k}, b_{k}\right]$ for $k \in \mathbf{N}$ and $\beta$ is 1-lipschitzian, $L_{\beta}(t) \leq 1$ for every $t \in[0, \infty)$ and $\beta$ is absolutely continuous on every closed interval $I \subset[0, \infty)$. We also see that there exists $m_{k} \geq p_{k}$, $m_{k} \in J$ for every $k \in \mathbf{N}$ such that $\gamma_{k m_{k}} \rightarrow \beta$.

We see that $a_{k} \rightarrow a, b_{k} \rightarrow b$ and let $a<a^{\prime}<b^{\prime}<b$. We can suppose that $a_{k}<a^{\prime}<b^{\prime}<b_{k}$ for every $k \in \mathbf{N}$. Let $k \in \mathbf{N}$ and $\gamma_{k m_{k}} \in \Gamma_{m_{k}}$. Then $\int_{\gamma_{k m_{k}}} \eta d s \leq$ $\int_{\gamma_{m k}} \eta d s \leq \rho$ for every $k \in \mathbf{N}$. Using Fatou's lemma and the lower semicontinuity of $\eta$, we find that

$$
\begin{aligned}
\int_{a^{\prime}}^{b^{\prime}} \eta(\beta(t)) L_{\beta}(t) d t & \leq \int_{a^{\prime}}^{b^{k}} \eta(\beta(t)) d t=\int_{a^{\prime}}^{b^{\prime}} \eta\left(\lim _{k \rightarrow \infty} \gamma_{k m_{k}}(t)\right) d t \leq \int_{a^{\prime}}^{b^{6}} \lim _{k \rightarrow \infty} \eta\left(\gamma_{k m_{k}}(t)\right) d t \\
& \leq \liminf _{k \rightarrow \infty} \int_{a^{\prime}}^{b^{\prime}} \eta\left(\gamma_{k m_{k}}(t) d t \leq \liminf _{k \rightarrow \infty} \int_{\gamma_{k m_{k}}} \eta d s \leq \rho<1 .\right.
\end{aligned}
$$

On the other side, since $\beta$ is absolutely continuous, we see from Lemma 2.2 that $\int_{a^{\prime}}^{b^{\prime}} \eta(\beta(t)) L_{\beta}(t) d t=\int_{\beta \mid\left[a^{\prime}, b^{\prime}\right]} \eta d s$. Letting $a^{\prime} \rightarrow a, b^{\prime} \rightarrow b$ and since $\beta \in \Gamma$, we find that

$$
1 \leq \int_{\beta} \eta d s=\int_{a}^{b} \eta(\beta(t)) L_{\beta}(t) d t \leq \rho<1 .
$$

We reached a contradiction and we showed that $\lambda \geq 1$.

Let now $K_{q} \subset \subset D$ be such that $K_{q} \nearrow D$ and $\Gamma_{m q}=\left\{\gamma \in \Gamma_{m} \mid \operatorname{Im} \gamma \subset K_{q}\right\}$ for $q \in \mathbf{N}$. Let $\rho_{m q} \in F\left(\Gamma_{m q}\right)$ be such that $\int_{X} \omega(x) \rho_{m q}(x)^{p} d \mu \leq M_{\omega}^{p}\left(\Gamma_{m q}\right)+\frac{1}{2^{m}}$ for every $q \in \mathbf{N}$. Since $\frac{\eta}{\lambda_{m}} \in F\left(\Gamma_{m}\right)$, we see that $\frac{1}{2}\left(\frac{\eta}{\lambda m}+\rho_{m q}\right) \in F\left(\Gamma_{m q}\right)$ for every $q \in \mathbf{N}$. Using Clarkson's inequality, we have

$$
\begin{aligned}
& \int_{X} \omega\left(\frac{1}{2}\left(\frac{\eta}{\lambda_{m}}+\rho_{m q}\right)\right)^{p} d \mu+\int_{X} \omega\left(\frac{1}{2}\left|\frac{\eta}{\lambda_{m}}-\rho_{m q}\right|\right)^{p} d \mu \\
& \leq \frac{1}{2}\left(\frac{1}{\lambda_{m}^{p}} \int_{X} \omega \eta^{p} d \mu+\int_{X} \omega \rho_{m q}^{p} d \mu\right)
\end{aligned}
$$

for every $q \in \mathbf{N}$. Then

$$
M_{\omega}^{p}\left(\Gamma_{m q}\right)+\int_{X} \omega\left(\frac{1}{2}\left|\frac{\eta}{\lambda_{m}}-\rho_{m q}\right|\right)^{p} d \mu
$$




$$
\begin{aligned}
& \leq \int_{X} \omega\left(\frac{1}{2}\left(\frac{\eta}{\lambda_{m}}+\rho_{m q}\right)\right)^{p} d \mu+\int_{X} \omega\left(\frac{1}{2}\left|\frac{\eta}{\lambda_{m}}-\rho_{m q}\right|\right)^{p} d \mu \\
& \leq \frac{1}{2}\left(\frac{1}{\lambda_{m}^{p}}\left(M_{\omega}^{p}(\Gamma)+\frac{\epsilon}{2}\right)+M_{\omega}^{p}\left(\Gamma_{m q}\right)+\frac{1}{2^{m}}\right)
\end{aligned}
$$

for every $q \in \mathbf{N}$.

Since $\frac{1}{m}<\frac{r}{4}$, we see from Lemma 2.8 that $M_{\omega}^{p}\left(\Gamma_{m q}\right) \leq\left(\frac{2}{r}\right)^{p} \int_{K_{q}} \omega(x) d \mu<\infty$ for every $q \in \mathbf{N}$. It results that

$$
0 \leq \int_{X} \omega\left(\frac{1}{2}\left|\frac{\eta}{\lambda_{m}}-\rho_{m q}\right|\right)^{p} d \mu \leq \frac{1}{2}\left(\frac{1}{\lambda_{m}^{p}}\left(M_{\omega}^{p}(\Gamma)+\frac{\epsilon}{2}\right)-M_{\omega}^{p}\left(\Gamma_{m q}\right)+\frac{1}{2^{m}}\right)
$$

for every $m, q \in \mathbf{N}$. Let $m_{\epsilon} \in \mathbf{N}$ be such that $\frac{1}{2^{m}}<\frac{\epsilon}{2 \lambda_{m}^{p}}$ for every $m \geq m_{\epsilon}$. Then $\lambda_{m}^{p} M_{\omega}^{p}\left(\Gamma_{m q}\right) \leq M_{\omega}^{p}(\Gamma)+\epsilon$ for every $m \geq m_{\epsilon}$ and every $q \in \mathbf{N}$.

Since $\Gamma_{m q} \nearrow \Gamma_{m}$, we use Ziemer's theorem to see that $M_{\omega}^{p}\left(\Gamma_{m q}\right) \nearrow M_{\omega}^{p}\left(\Gamma_{m}\right)$ if $q \rightarrow \infty$ and then $\lambda_{m}^{p} M_{\omega}^{p}\left(\Gamma_{m}\right) \leq M_{\omega}^{p}(\Gamma)+\epsilon$ for $m \geq m_{\epsilon}$. Letting $m \rightarrow \infty$, we find that $\lim _{m \rightarrow \infty} M_{\omega}^{p}\left(\Gamma_{m}\right) \leq M_{\omega}^{p}(\Gamma)+\epsilon$ and letting $\epsilon \rightarrow 0$ we find that $\lim _{m \rightarrow \infty} M_{\omega}^{p}\left(\Gamma_{m}\right) \leq$ $M_{\omega}^{p}(\Gamma)$. We finally proved that $\lim _{m \rightarrow \infty} M_{\omega}^{p}\left(\Gamma_{m}\right)=M_{\omega}^{p}(\Gamma)$.

Proof of Lemma 2.5. Suppose that $M_{\omega}^{p}(x)=0$ and let $b_{0}>0$ be such that $\bar{B}\left(x, b_{0}\right)$ is compact. Let $0<b<b_{0}, C_{0}=\{x\}, C_{1}=S(x, b)$ and let $0<r<b$ and $\Gamma_{r}=\Gamma_{x, r, b}$. We see form the preceding theorem that $\lim _{r \rightarrow 0} M_{\omega}^{p}\left(\Gamma_{r}\right)=M_{\omega}^{p}(x)=0$.

Let now $\Gamma$ be the family of all nonconstant paths having at least a limit point in $x$. Let $\Gamma_{j}=\left\{\gamma:[0,1) \rightarrow D\right.$ path $\mid x$ is a limit point of $\gamma$ and $\left.d(\operatorname{Im} \gamma) \geq \frac{1}{j}\right\}$ for $j \in \mathbf{N}$. Let $j \in \mathbf{N}$ be fixed and let $0<r_{k}<\frac{1}{j}, r_{k} \rightarrow 0$. Then $\Gamma_{j}>\Gamma_{x, r_{k}, \frac{1}{j}}$ and hence $M_{\omega}^{p}\left(\Gamma_{j}\right) \leq M_{\omega}^{p}\left(\Gamma_{x, r_{k}, \frac{1}{j}} \rightarrow 0\right.$ if $k \rightarrow \infty$. Since $M_{\omega}^{p}\left(\Gamma_{j}\right)=0$ for every $j \in \mathbf{N}$ and $\Gamma=\bigcup_{j=1}^{\infty} \Gamma_{j}$ we see that $M_{\omega}^{p}(\Gamma)=0$ and hence $M_{\omega}^{p}(x)=0$ if $\lim _{r \rightarrow 0} M_{\omega}^{p}\left(\Gamma_{x, r, b}\right)=0$ for every fixed $0<b<d(x, \partial D)$.

\section{Proofs of the results}

Proof of Theorem 1.1. Suppose that $f$ is not continuous at $x$. We can find $x_{j} \rightarrow x, y_{j} \rightarrow x, x_{j}, y_{j} \notin E$ for every $j \in \mathbf{N}$ such that $f\left(x_{j}\right) \rightarrow b_{1}, f\left(y_{j}\right) \rightarrow b_{2}$ with $b_{1}, b_{2} \in \bar{Y}, b_{1} \neq b_{2}$. Let $0<r_{j}<r_{x}, r_{j} \rightarrow 0$ such that there exists $U_{j} \in \mathcal{V}(x)$ pathwise connected such that $\bar{U}_{j} \subset B\left(x, r_{j}\right)$ and $x_{j}, y_{j} \in U_{j}$ for every $j \in \mathbf{N}$. Since $E$ is nowhere disconnecting, we can find a path $H_{j}$ joining $x_{j}$ with $y_{j}$ in $U_{j} \backslash E$ for every $j \in \mathbf{N}$. We have two cases.

Case 1. There exists $y \in Y$ and $R>0$ and an infinite set $J_{1} \subset \mathbf{N}$ such that $f\left(H_{j}\right) \cup M \subset B(y, R)$ for every $j \in J_{1}$. Then $b_{1}, b_{2} \in B(y, R)$ and $d\left(f\left(H_{j}\right)\right) \geq$ $d\left(b_{1}, b_{2}\right)$ for every $j \in J_{1}$. Let $\rho_{1}=C_{1} R^{Q-q-1} \min \left\{d\left(b_{1}, b_{2}\right), d(M)\right\}$ and let $\Gamma_{j}^{\prime}=$ $\Delta\left(f\left(H_{j}\right), M, Y\right)$ for $j \in J_{1}$. Using Theorem A, we see that $M_{q}\left(\Gamma_{j}^{\prime}\right) \geq \rho_{1}$ for every $j \in J_{1}$.

Case 2. There exists $y \in Y$ and $R>0$ and an infinite set $J_{2} \subset \mathbf{N}$ such that $M \subset B(y, R)$ and a subpath $Q_{j} \subset H_{j}$ such that $Q_{j} \cap B(y, R) \neq \phi, Q_{j} \cap B(y, 2 R) \neq \phi$, $Q_{j} \subset B(y, 3 R)$ for every $j \in J_{2}$. Let $\rho_{2}=C_{1}(3 R)^{Q-q-1} \min \{R, d(M)\}$ and let $\Gamma_{j}^{\prime}=\Delta\left(f\left(Q_{j}\right), M, Y\right)$ for $j \in J_{2}$. Then $M_{q}\left(\Gamma_{j}^{\prime}\right) \geq \rho_{2}$ for every $j \in J_{2}$ and $\mathbf{N}=J_{1} \cup J_{2}$. Let $\rho=\min \left\{\rho_{1}, \rho_{2}\right\}$. We proved that $M_{q}\left(\Gamma_{j}^{\prime}\right) \geq \rho$ for every $j \in \mathbf{N}$. 
Let $j \in \mathbf{N}$ be fixed and let $\Gamma_{j}$ be the family of all maximal liftings of some paths from $\Gamma_{j}^{\prime}$ starting from some point of $H_{j}$ or $Q_{j}$. We can suppose that $\bar{B}\left(x, r_{x}\right)$ is compact and let $p:[0,1] \rightarrow Y \backslash M, p \in \Gamma_{j}^{\prime}$. Using the compactness of $\bar{B}\left(x, r_{x}\right)$ and the openness of the mapping $f$, we see that if $q:[0, a) \rightarrow X$ is a maximal lifting of the path $p$ from some point of $H_{j}$ or $Q_{j}$, then either the open path $q:[0, a) \rightarrow X$ has at least a limit point in $\partial\left(B\left(x, r_{x}\right) \backslash E\right) \subset E \cup S\left(x, r_{x}\right)$, or intersects $S\left(x, r_{x}\right)$. Let $\Gamma_{1 j}=$ $\left\{\alpha \in \Gamma_{j} \mid \alpha\right.$ has at least a limit point in $\left.E\right\}$ and $\Gamma_{2 j}=\left\{\alpha \in \Gamma_{j} \mid \operatorname{Im} \alpha \cap S\left(x, r_{x}\right) \neq \phi\right\}$ and $\Delta_{j}=\Gamma_{x, r_{j}, r_{x}}$. Then $\Gamma_{j}=\Gamma_{1 j} \cup \Gamma_{2 j}, \Gamma_{j}^{\prime}>f\left(\Gamma_{j}\right), M_{\omega}^{p}\left(\Gamma_{1 j}\right)=0, \Gamma_{2 j}>\Delta_{j}$. Since $M_{\omega}^{p}(x)=0$, we see from Lemma 2.5 that $\lim _{j \rightarrow \infty} M_{\omega}^{p}\left(\Delta_{j}\right)=0$. We have

$$
\begin{aligned}
\rho & \leq M_{q}\left(\Gamma_{j}^{\prime}\right) \leq M_{q}\left(f\left(\Gamma_{j}\right)\right) \leq M_{q}\left(f\left(\Gamma_{1 j} \cup f\left(\Gamma_{2 j}\right)\right) \leq M_{q}\left(f\left(\Gamma_{1 j}\right)\right)+M_{q}\left(f\left(\Gamma_{2 j}\right)\right)\right. \\
& \leq \gamma\left(M_{\omega}^{p}\left(\Gamma_{1 j}\right)\right)+\gamma\left(M_{\omega}^{p}\left(\Gamma_{2 j}\right)\right)=\gamma\left(M_{\omega}^{p}\left(\Gamma_{2 j}\right)\right) \leq \gamma\left(M_{\omega}^{p}\left(\Delta_{j}\right)\right) \rightarrow 0
\end{aligned}
$$

if $j \rightarrow \infty$. We reached a contradiction and hence there exists $\lim _{y \rightarrow x} f(y)=l \in$ $\bar{Y}$.

Proof of Theorem 1.2. It results immediately from Theorem 1.1.

Proof of Theorem 1.3. Suppose that the family $W$ is not equicontinuous at $x$. Then there exists $\epsilon>0, r_{j} \rightarrow 0, y_{j} \in B\left(x, r_{j}\right)$ such that $d\left(f_{j}\left(y_{j}\right), f_{j}(x)\right)>\epsilon$ for every $j \in \mathbf{N}$. Since each mapping $f_{j}$ is continuous at $x$, we can find $x_{j} \neq x$ such that $d\left(f_{j}\left(y_{j}\right), f_{j}\left(x_{j}\right)\right) \geq \epsilon$, with $y_{j} \neq x, x_{j} \neq x$ for every $j \in \mathbf{N}$ and let $H_{j}$ be a path joining $y_{j}$ with $x_{j}$ in $B\left(x, r_{j}\right) \backslash\{x\}$ for $j \in \mathbf{N}$. Then $d\left(f_{j}\left(H_{j}\right)\right) \geq d\left(f_{j}\left(y_{j}\right), f_{j}\left(x_{j}\right)\right) \geq \epsilon$, $M_{f_{j}} \cap B\left(y, R_{0}\right) \neq \phi$ and we can suppose that $f_{j}\left(H_{j}\right) \cap B\left(y, R_{0}\right) \neq \phi$ for every $j \in \mathbf{N}$. We have 4 cases.

Case 1. There exists $R>R_{0}$ and an infinite set $J_{1} \subset \mathbf{N}$ such that $f_{j}\left(H_{j}\right) \cup M_{f_{j}} \subset$ $B(y, R)$ for every $j \in J_{1}$. Let $\rho_{1}=C_{1} R^{Q-q-1} \min \{\epsilon, \delta\}$ and $\Gamma_{j}^{\prime}=\Delta\left(f_{j}\left(H_{j}\right), M_{f_{j}}, Y\right)$ for $j \in J_{1}$. We see from Theorem A that $M_{q}\left(\Gamma_{j}^{\prime}\right) \geq \rho_{1}$ for every $j \in J_{1}$.

Case 2. There exists $R>R_{0}$ and an infinite set $J_{2} \subset \mathbf{N}$ such that $f_{j}\left(H_{j}\right) \subset$ $B(y, R), M_{f_{j}} \cap B(y, R) \neq \phi, M_{f_{j}} \cap \complement \bar{B}(y, 3 R) \neq \phi$ for every $j \in J_{2}$. Using Lemma 2.8, we find a continuum $K_{j} \subset M_{f_{j}} \cap \bar{C}(y, R, 3 R)$ such that $d\left(K_{j}\right) \geq R$ for every $j \in J_{2}$. Let $\rho_{2}=C_{1}(3 R)^{Q-q-1} \min \{\epsilon, R\}$ and let $\Gamma_{j}^{\prime}=\Delta\left(f_{j}\left(H_{j}\right), K_{j}, Y\right)$ for $j \in J_{2}$. Then $M_{q}\left(\Gamma_{j}^{\prime}\right) \geq \rho_{2}$ for $j \in J_{2}$.

Case 3. There exists $R>R_{0}$ and an infinite set $J_{3} \subset \mathbf{N}$ such that $M_{f_{j}} \subset B(y, R)$ and a subpath $Q_{j}$ of $H_{j}$ such that $Q_{j} \cap B(y, R) \neq \phi, Q_{j} \cap B(y, 2 R) \neq \phi, Q_{j} \subset B(y, 3 R)$ for $j \in J_{3}$. Let $\rho_{3}=C_{1}(3 R)^{Q-q-1} \min \{R, \delta\}$ and let $\Gamma_{j}^{\prime}=\Delta\left(f_{j}\left(Q_{j}\right), M_{f_{j}}, Y\right)$ for $j \in J_{3}$. We see from Theorem A that $M_{q}\left(\Gamma_{j}^{\prime}\right) \geq \rho_{3}$ for every $j \in J_{3}$.

Case 4. There exists $R>R_{0}$ and an infinite set $J_{4} \subset \mathbf{N}$ such that we find a subpath $Q_{j}$ of $H_{j}$ such that $Q_{j} \cap B(y, R) \neq \phi, Q_{j} \cap B(y, 2 R) \neq \phi, Q_{j} \subset B(y, 3 R)$ and a continuum $K_{j} \subset M_{f_{j}} \cap \bar{C}(y, R, 3 R)$ and $d\left(K_{j}\right) \geq R$ for every $j \in J_{4}$. Let $\rho_{4}=C_{1}(3 R)^{Q-q}$ and $\Gamma_{j}^{\prime}=\Delta\left(f_{j}\left(Q_{j}\right), K_{j}, Y\right)$ for $j \in J_{4}$. Using Theorem A, we see that $M_{q}\left(\Gamma_{j}^{\prime}\right) \geq \rho_{4}$ for every $j \in J_{4}$. Let $\rho=\min \left\{\rho_{1}, \rho_{2}, \rho_{3}, \rho_{4}\right\}$ and we see that $\mathbf{N}=J_{1} \cup J_{2} \cup J_{3} \cup J_{4}$.

Let $\Gamma_{j}$ be the family of all maximal liftings of some paths from $\Gamma_{j}^{\prime}$ starting from some points of the sets $H_{j}$ or $Q_{j}$ for $j \in \mathbf{N}$. We can suppose that $\bar{B}\left(x, r_{x}\right)$ is compact and that $0<r_{j}<r_{x}$ for $j \in \mathbf{N}$. Let $\Gamma_{1 j}=\left\{\alpha \in \Gamma_{j} \mid \alpha\right.$ has at least a limit point in $\left.x\right\}$ and $\Gamma_{2 j}=\left\{\alpha \in \Gamma_{j} \mid \operatorname{Im} \alpha \cap B\left(x, r_{x}\right) \neq \phi\right\}$ for $j \in \mathbf{N}$. Then $\Gamma_{j}=\Gamma_{1 j} \cup \Gamma_{2 j}, \Gamma_{j}^{\prime}>f_{j}\left(\Gamma_{j}\right)$ and $\Gamma_{2 j}>\Gamma_{x, r_{j}, r_{x}}$ for every $j \in \mathbf{N}$. Let $\beta_{j}=d\left(x, H_{j}\right)>0$ for $j \in \mathbf{N}$ and let us fix 
$j \in \mathbf{N}$. Let $0<\alpha_{j k}<\beta_{j}, \alpha_{j k} \rightarrow 0$. Then $\Gamma_{1 j}>\Gamma_{x, \alpha_{j k}, \beta_{j}}$ for every $k \in \mathbf{N}$. We have

$$
\begin{aligned}
0 & <\rho<M_{q}\left(\Gamma_{j}^{\prime}\right) \leq M_{q}\left(f_{j}\left(\Gamma_{j}\right)\right)=M_{q}\left(f_{j}\left(\Gamma_{1 j}\right) \cup f_{j}\left(\Gamma_{2 j}\right)\right) \leq M_{q}\left(f_{j}\left(\Gamma_{1 j}\right)\right)+M_{q}\left(f_{j}\left(\Gamma_{2 j}\right)\right) \\
& \leq M_{q}\left(f_{j}\left(\Gamma_{x, \alpha_{j k}, \beta_{j}}\right)\right)+M_{q}\left(f_{j}\left(\Gamma_{x, r_{j}, r_{x}}\right)\right) \leq \gamma\left(\Delta_{\omega}^{p}\left(\Gamma_{x, \alpha_{j k}, \beta_{j}}\right)\right)+\gamma\left(\Delta_{\omega}^{p}\left(\Gamma_{x, r_{j}, r_{x}}\right)\right) .
\end{aligned}
$$

Letting $k \rightarrow \infty$, we find that

$$
0<\rho<M_{q}\left(\Gamma_{j}^{\prime}\right) \leq \gamma\left(\Delta_{\omega}^{p}\left(\Gamma_{x, r_{j}, r_{x}}\right)\right)
$$

for every $j \in \mathbf{N}$. Letting now $j \rightarrow \infty$ and using the fact that $\gamma\left(\Delta_{\omega}^{p}\left(\Gamma_{x, r_{j}, r_{x}}\right)\right) \rightarrow 0$ we reached a contradiction. We finally proved that the family $W$ is equicontinuous at $x$.

Proof of Theorem 1.4. Suppose that there exist a continuum $M \subset Y$ with Card $M>1$ such that $f(X \backslash E) \subset Y \backslash M$. Let $K \subset B(x, r) \backslash E$ be compact, connected such that Card $K>1$. Then $f(K)$ is a continuum and Card $f(K)>1$ and let $y \in Y$ and $R_{0}>0$ be such that $f(K) \cup M \subset B\left(y, R_{0}\right)$. Let $R>R_{0}$ and $\Gamma^{\prime}=$ $\Delta(f(K), M, Y)$ and let $\Gamma$ be the family of all maximal liftings of some paths from $\Gamma^{\prime}$ starting from some points in $K$. Let $\Gamma_{1}=\{\alpha \in \Gamma \mid \alpha$ has at least a limit point in $E\}$ and $\Gamma_{2}=\{\alpha \in \Gamma \mid \operatorname{Im} \alpha \cap S(y, R) \neq \phi\}$. Then $\Gamma=\Gamma_{1} \cup \Gamma_{2}, \Gamma^{\prime}>f(\Gamma), M_{\omega}^{p}\left(\Gamma_{1}\right)=0$, $\Gamma_{2}>\Gamma_{x, r, R}$ and let $\delta=C_{1} R_{0}^{Q-q+1} \min \{d(f(K)), M\}$. We have

$$
\begin{gathered}
0<\delta \leq M_{q}\left(\Gamma^{\prime}\right) \leq M_{q}(f(\Gamma)) \leq M_{q}\left(f\left(\Gamma_{1} \cup \Gamma_{2}\right)\right) \leq M_{q}\left(f\left(\Gamma_{1}\right)\right)+M_{q}\left(f\left(\Gamma_{2}\right)\right) \leq \\
\leq \gamma\left(M_{\omega}^{p}\left(\Gamma_{1}\right)\right)+\gamma\left(M_{\omega}^{p}\left(\Gamma_{2}\right)\right)=\gamma\left(M_{\omega}^{p}\left(\Gamma_{2}\right)\right) \leq \gamma\left(M_{\omega}^{p}\left(\Gamma_{x, r, R}\right)\right) \leq \gamma\left(\Delta_{\omega}^{p}\left(\Gamma_{x, r, R}\right)\right) \rightarrow 0
\end{gathered}
$$

if $R \rightarrow \infty$. We reached a contradiction and hence $\operatorname{dim}(Y \backslash f(X \backslash E))=0$.

Proof of Theorem 1.5. Let $U_{k} \in \mathcal{V}(x)$ be connected such that $\bar{U}_{k} \subset B\left(x, \frac{1}{k}\right)$ and is compact, $\partial U_{k} \cap E=\phi$ for every $k \in \mathbf{N}$ and suppose that $z \notin \bigcap_{k=1}^{\infty} f\left(B\left(x, \frac{1}{k}\right) \cap D\right)$. Let $F_{k}=C\left(f, x, \bar{B}\left(x, \frac{1}{k}\right) \cap(\partial D \backslash E) \backslash\{x\}\right)$ for $k \in \mathbf{N}$. Then $F_{k+1} \subset F_{k}$ for every $k \in \mathbf{N}$ and $C(f, x, \partial D \backslash E)=\bigcap_{k=1}^{\infty} \bar{F}_{k}$. Let $2 \alpha=d(z, C(f, x, \partial D \backslash E)$. We can suppose that $\alpha<d\left(z, \bar{F}_{k}\right)$ for every $k \in \mathbf{N}$.

Let $\rho_{k}=d\left(z, f\left(\partial U_{k} \cap D\right)\right)$ for $k \in \mathbf{N}$. Suppose that there exists $k_{p} \in \mathbf{N}$ such that $\rho_{k_{p}}=0$ for every $p \in \mathbf{N}$. Let $p \in \mathbf{N}$ be fixed. We can find $a_{k_{p} j} \in \partial U_{k_{p}} \cap D$ such that $f\left(a_{k_{p} j}\right) \rightarrow z$ and if necessarily extracting a subsequence, we can presume that there exists $a_{p} \in \partial U_{k_{p}} \cap \bar{D}$ such that $a_{k_{p} j} \rightarrow a_{p}$. If $a_{p} \in \partial U_{k_{p}} \cap \partial D$, then $a_{p} \in \partial U_{k_{p}} \cap(\partial D \backslash E)$ and then $z \in \bar{F}_{k_{p}}$, which contradicts the fact that $d\left(z, \bar{F}_{k_{p}}\right)=\alpha>0$. We find that $a_{p} \in \partial U_{k_{p}} \cap D \subset B\left(x, \frac{1}{k_{p}}\right) \cap D$ and $f\left(a_{p}\right)=z$ for every $p \in \mathbf{N}$ and this contradicts the fact that $z \notin \bigcap_{p=1}^{\infty} f\left(B\left(x, \frac{1}{k_{p}}\right) \cap D\right)$.

We proved that there exists $k_{o} \in \mathbf{N}$ such that $\rho_{k}>0$ for every $k \geq k_{0}$ and we can suppose that $\rho_{k}>0$ for every $k \in \mathbf{N}$. Since $z \in C(f, x)$, there exists $\alpha_{k} \in U_{k} \cap D$ such that $f\left(\alpha_{k}\right) \rightarrow z$. Let $C$ be the constant from property $\left(P_{q}\right)$ corresponding to the point $z$. Since $f$ is an open mapping, there exist $0<r_{k}<\frac{1}{C} \rho_{k}$ and open subsets $C_{0}$ in $S\left(z, r_{k}\right)$ and $Q_{0} \subset U_{k} \cap D$ such that $f\left(Q_{0}\right)=C_{0}$.

Let $k \in \mathbf{N}$ be fixed. Let $\varphi_{0}=r_{k}$ and $\varphi_{j} \searrow 0$. Let $A_{1}=\left\{y \in S\left(z, \varphi_{1}\right) \mid\right.$ for every path $p:[0,1] \rightarrow B\left(z, C \varphi_{0}\right)$ with $p(0) \in C_{0}, p(1)=y$ there exists $\alpha \in Q_{0}$ such that $f(\alpha)=p(0)$ and we cannot lift $p$ from $\alpha$. Suppose that there exists a continuum $Q \subset A_{1}$ with $\operatorname{Card} Q>1$. Let $\Gamma^{\prime}=\Delta\left(C_{0}, Q, B\left(z, C \varphi_{0}\right)\right)$. Since $\operatorname{dim} C_{0} \geq 1$ there exists a continuum $M_{0} \subset C_{0}$ with Card $M_{0}>1$ and we see from Theorem A that $0<M_{q}\left(\Gamma^{\prime}\right)$.

Let $\Gamma$ be the family of all maximal liftings of some paths $p:[0,1] \rightarrow Y$ from $\Gamma^{\prime}$ such that there exists a point $b \in Q_{0}$ such that $f(b)=p(0)$ and we cannot lift $p$ from $\left.b\right\}$. 
Let $p:[0,1] \rightarrow B\left(z, C r_{k}\right), p \in \Gamma^{\prime}$ and let $q:[0, a) \rightarrow D$ be a maximal lifting of $p$ from some point $b \in C_{0}$ with $f(b)=p(0)$ and $0<a \leq 1$. We see that $\overline{D \cap U_{k}}$ is compact, that $\complement \overline{D \cap U_{k}}$ is nonempty and open and $\operatorname{Im} q=\left(\operatorname{Im} q \cap D \cap U_{k}\right) \cup\left(\operatorname{Im} q \cap \complement \overline{D \cap U_{k}}\right) \cup$ $\left(\operatorname{Im} q \cap \partial\left(D \cap U_{k}\right)\right)$. Since $\operatorname{Im} q$ is connected, we see that if $\operatorname{Im} q \cap C \overline{D \cap U_{k}} \neq \phi$, then $\operatorname{Im} q \cap \partial\left(D \cap U_{k}\right) \neq \phi$. Since $\partial\left(D \cap U_{k}\right) \subset\left(\bar{U}_{k} \cap \partial D\right) \cup\left(D \cap \partial U_{k}\right)$ and $\operatorname{Im} q \subset D$, it results that $\operatorname{Im} q \cap \partial U_{k} \neq \phi$ and hence there exists $0<c<a$ such that $q(c) \in \partial U_{k}$ and then $p(c)=f(q(c)) \in f\left(D \cap \partial U_{k}\right)$. We reached a contradiction, since $d(p(c), z)<C r_{k}<\rho_{k}$ and on the otherside $d(p(c), z) \geq d\left(z, f\left(\partial U_{k} \cap D\right)=\rho_{k}\right.$.

We proved that $\operatorname{Im} q \subset D \cap U_{k}$. Let $t_{n} \nearrow a$. Since $\overline{D \cap U_{k}}$ is compact and if necessarily extracting a subsequence, we can suppose that there exists $w \in \overline{D \cap U_{k}}$ such that $q\left(t_{n}\right) \rightarrow w$. If $w \in D \cap U_{k}$, we use the openness of the mapping $f$ and we find $U \in \mathcal{V}(w), \bar{U} \subset D \cap U_{k}$ such that $p(a)=f(q(a)) \in f(U) \subset f\left(D \cap U_{k}\right)$ and this contradicts the maximality of the open path $q:[0, a) \rightarrow D \cap U_{k}$. It results that $w \in \partial\left(D \cap U_{k}\right) \subset\left(\bar{U}_{k} \cap \partial D\right) \cup\left(D \cap \partial U_{k}\right)$. If $w \in D \cap \partial U_{k}$, then $p(a)=$ $\lim _{n \rightarrow \infty} p\left(t_{n}\right)=\lim _{n \rightarrow \infty} f\left(q\left(t_{n}\right)\right)=f(w)$ and $d(p(a), z)<C r_{k}<\rho_{k}$ and on the other side $d(p(a), z) \geq d\left(z, f\left(D \cap \partial U_{k}\right)\right)=\rho_{k}$ and we reached a contradiction.

If $w \in \partial D \backslash E$, then $z \in \bar{F}_{k}$ and on the other side $d\left(z, \bar{F}_{k}\right)=\alpha>0$. It results that $w \in \partial D \cap E$. We see that $M_{\omega}^{p}(E)=0$ and hence $M_{\omega}^{p}(\Gamma)=0$ and since $\Gamma^{\prime}>f(\Gamma)$, we have $0<M_{q}\left(\Gamma^{\prime}\right) \leq M_{q}(f(\Gamma)) \leq \gamma\left(M_{\omega}^{p}(\Gamma)\right)=0$ and we reached a contradiction. We proved that $\operatorname{dim} A_{1}=0$.

Let us show that $A_{1}$ is closed in $S\left(z, \varphi_{1}\right)$. Let $y_{n} \in A_{1}, y_{n} \rightarrow y \in S\left(z, \varphi_{1}\right)$ and suppose that $y \notin A_{1}$. Then there exists $\alpha \in Q_{0}$, a path $\beta$ : $[0,1] \rightarrow B\left(z, C \varphi_{0}\right)$ with $\beta(0)=f(\alpha), \beta(1)=y$ and a path $\gamma:[0,1] \rightarrow D \cap U_{k}$ such that $\gamma(0)=\alpha$ and $\beta=f \circ \gamma$. Let $V_{1}, \ldots, V_{m}$ be normal domains such that $\operatorname{Im} \gamma \subset \bigcup_{i=1}^{m} V_{i}, f\left(\bar{V}_{i}\right) \subset B\left(z, C \varphi_{0}\right)$, $i=1, \ldots, m$ and there exists points $z_{i} \in V_{i} \cap V_{i+1}$ for $i=1, \ldots, m-1$ and let $y_{n} \in f\left(V_{m}\right)$ for some $n \in \mathbf{N}$. We choose paths $q_{i}:[0,1] \rightarrow V_{i}, i=1, \ldots, m$ such that $q_{1}(0)=\alpha, q_{1}(1)=z_{1}, q_{i+1}(0)=q_{i}(1)=z_{i}$ for $i=1, \ldots, m-1$ and $f\left(q_{m}(1)\right)=y_{n}$. Let $q=q_{1} \vee, \ldots, \vee q_{m}$ and $p=f \circ q$. Then $q(0)=\alpha, p(0)=f(\alpha), p=f \circ q, p(1)=y_{n}$, $\operatorname{Im} p \subset B\left(z, C \varphi_{0}\right)$ and we can lift $p$ from $\alpha$. This contradicts the fact that $y_{n} \in A_{1}$. We proved that $A_{1}$ is closed in $S\left(z, \varphi_{1}\right)$.

Let $\Delta_{1}^{\prime}=\Delta\left(f\left(Q_{0}\right), S\left(z, \varphi_{1}\right) \backslash A_{1}, B\left(z, C \varphi_{0}\right)\right)$ and let $Q_{1}$ be the set of all endpoints of the maximal liftings of the paths from $\Delta_{1}^{\prime}$ starting from some points in $Q_{0}$. We see that if $p:[0,1] \rightarrow B\left(z, C \varphi_{0}\right)$ is a path from $\Delta_{1}^{\prime}$ and $p(0)=f(\alpha)$ for some $\alpha \in Q_{0}$, there always exists a path $q:[0,1] \rightarrow D \cap U_{k}$ such that $q(0)=\alpha$ and $f \circ q=p$. We also see that $f\left(Q_{1}\right)=S\left(z, \varphi_{1}\right) \backslash A_{1}$.

Let $A_{2}=\left\{y \in S\left(z, \varphi_{2}\right) \mid\right.$ for every path $p:[0,1] \rightarrow B\left(z, C \varphi_{1}\right)$ with $p(0) \in f\left(Q_{1}\right)$ and $p(1)=y$ there exists a point $b \in Q_{1}$ with $p(0)=f(b)$ and we cannot lift $p$ from $b\}$. As before, we see that $\operatorname{dim} A_{2}=0$ and that $A_{2}$ is closed in $S\left(z, \varphi_{2}\right)$.

Let $\left.\Delta_{2}^{\prime}=\Delta\left(f\left(Q_{1}\right)\right), S\left(z, \varphi_{2}\right) \backslash A_{2}, B\left(z, C \varphi_{1}\right)\right)$ and let $Q_{2}$ be the set of all endpoints of the maximal liftings of some paths from $\Delta_{2}^{\prime}$ starting from some points in $Q_{1}$. We see that if $p:[0,1] \rightarrow B\left(z, C \varphi_{1}\right)$ is a path from $\Delta_{2}^{\prime}$ and $p(0)=f(b)$ with $b \in Q_{1}$, there always exists a path $q:[0,1] \rightarrow D \cap U_{k}$ such that $q(0)=b$ and $f \circ q=p$.

We also remark that if $p_{1}:[0,1] \rightarrow B\left(z, C \varphi_{0}\right)$ is a path from $\Delta_{1}^{\prime}$ and $b_{0} \in Q_{0}$ is such that $f\left(b_{0}\right)=p_{1}(0)$, there exists $y_{1} \in f\left(Q_{0}\right)=B\left(z, \varphi_{1}\right) \backslash A_{1}$ such that $p_{1}(1)=y_{1}$ and a path $q_{1}:[0,1] \rightarrow D \cap U_{k}$ such that $q_{1}(0)=b_{0}$ and $f \circ q_{1}=p_{1}$. If $p_{2}:[0,1] \rightarrow$ $B\left(z, C \varphi_{1}\right)$ is a path from $\Delta_{2}^{\prime}$ such that $p_{2}(0)=y_{1}$ and $p_{2}(1) \in S\left(z, \varphi_{z}\right) \backslash A_{2}$, there exists a path $q_{2}:[0,1] \rightarrow D \cap U_{k}$ such that $q_{2}(0)=q_{1}(1)$ and $f\left(q_{2}(0)\right)=f\left(q_{1}(1)\right)=$ $p_{1}(1)=y_{1}$ and $f \circ q_{2}=p_{2}$. In this way we find closed sets $A_{j}$ in $S\left(z, \varphi_{j}\right)$ with $\operatorname{dim} A_{j}=0$ and sets $Q_{j} \subset D \cap U_{k}$ with $f\left(Q_{j}\right)=S\left(z, \varphi_{j}\right) \backslash A_{j}$ for $j \geq 1$ and such that 
for every path $p_{j}:[0,1] \rightarrow B\left(z, C \varphi_{j-1}\right)$ with $p_{j}(0) \in f\left(Q_{j-1}\right), p_{j}(1) \in f\left(Q_{j}\right)$, there exists a path $q_{j}:[0,1] \rightarrow D \cap U_{k}$ with $q_{j}(0) \in Q_{j-1}, q_{j}(0)=q_{j-1}(1), q_{j}(1) \in Q_{j}$ and $f \circ q_{j}=p_{j}$ for every $j \geq 1$.

Let $\tilde{p}_{k}:[0, \infty) \rightarrow B\left(z, C \varphi_{0}\right), \tilde{p}_{k}=p_{1} \vee p_{2} \vee, \ldots, \vee p_{n} \vee, \ldots$ and $\tilde{q}_{k}:[0, \infty) \rightarrow D \cap$ $U_{k}, \tilde{q}_{k}=q_{1} \vee q_{2} \vee, \ldots, \vee q_{n} \vee, \ldots$, . Then $\tilde{p}_{k}$ and $\tilde{q}_{k}$ are open paths and $\lim _{t \rightarrow \infty} \tilde{p}_{k}(t)=z$, $f \circ \tilde{q}_{k}=\tilde{p}_{k}$ and $\tilde{q}_{k}(0) \in Q_{0}$.

Let $B_{k}$ be the set of all limit points of the open path $\tilde{q}_{k}:[0, \infty) \rightarrow D \cap U_{k}$. Then $B_{k}$ is compact, connected. Suppose that $\operatorname{Card}\left(B_{k} \cap U_{k} \cap D\right)>1$. We use Lemma 2.8 and we find a continuum $K_{k} \subset B_{k} \cap U_{k} \cap D$ with Card $K_{k}>1$ and since $f(a)=z$ for every $a \in K_{k}$ and $f$ is a light mapping, we reached a contradiction. We have 3 possible cases.

Case 1. $B_{k}=\left\{x_{k}\right\}$ with $x_{k} \in \bar{U}_{k} \cap D$ and $f\left(x_{k}\right)=z$. Since $z \notin \bigcap_{r>0} f(B(x, r) \cap$ $D$ ) we see that case 1 cannot hold for infinitely many $k \in \mathbf{N}$. We can suppose that case 1 does not hold for every $k \in \mathbf{N}$.

Case 2. Card $B_{k}>1$ and hence $B_{k} \subset \bar{U}_{k} \cap \partial D$. Since $\operatorname{dim} B_{k} \geq 1$ and $\operatorname{dim} E=0$, we can find a point $x_{k} \in \bar{U}_{k} \cap(\partial D \backslash E)$ and $t_{n p} \rightarrow \infty$ such that $\tilde{q}_{k}\left(t_{n p}\right) \rightarrow x_{k}$. Then $f\left(\tilde{q}_{k}\left(t_{n p}\right)=\tilde{p}_{k}\left(t_{n p}\right) \rightarrow z\right.$ and this implies that $z \in C\left(f, x_{k}\right) \subset F_{k}$. We reached a contradiction, since $d\left(z, F_{k}\right)>\alpha>0$. We proved that case 2 cannot hold.

Case 3. $B_{k}=\left\{x_{k}\right\}$ with $x_{k} \in \bar{U} \cap D$. Since $\lim _{t \rightarrow \infty} \tilde{q}_{k}(t)=x_{k}$, we prove as before that we cannot find infinitely many $k \in \mathbf{N}$ such that $x_{k} \in \bar{U}_{k} \cap(\partial D \backslash E)$. We can suppose that $x_{k} \in E$ for every $k \in \mathbf{N}$ and hence $x_{k} \rightarrow x$ and $z \in A\left(f, x_{k}\right)$ for every $x \in \mathbf{N}$.

Proof of Theorem 1.6. Since $x \in \operatorname{Int} D$, we see that $B(x, r) \cap \partial D=\phi$ for small $r>0$ and hence $C(f, x, \partial D \backslash E)=\phi$, Since $x$ is an essential singularity of $f$, we see from Theorem 1.2 that $\operatorname{dim} Y \backslash(f(B(x, r) \backslash E))=0$ for every $r>0$ and since $\operatorname{dim} Y \geq 1$, we see that $C(f, x)=Y$. We apply now Theorem 1.5. It is obvious that if $x$ is an isolated essential singularity of $f$, we can use condition (1.4) instead of condition (1.3).

Proof of Theorem 1.7. Suppose that $Y \neq \bar{Y}$ and that there exists $b \in B$ and $\epsilon>0$ such that $M_{\omega}^{p}(E \cap B(b, \epsilon))=0$. Since $M_{\omega}^{p}\left(B \cap B\left(b, \frac{\epsilon}{2}\right)\right)>0$, there exists a point $y \in(B \backslash E) \cap B\left(b, \frac{\epsilon}{2}\right)$. It results that there exists a path $\alpha:[0,1) \rightarrow D$ such that $\lim _{t \rightarrow 1} \alpha(t)=y$ and $\lim _{t \rightarrow 1} f(\alpha(t))$ in $\bar{Y}$ does not exist. There exists $y_{1}, y_{2} \in \bar{Y}$, $y_{1} \neq y_{2}$ and $t_{j} \nearrow 1$ such that $f\left(\alpha\left(t_{2 j}\right)\right) \rightarrow y_{1}, f\left(\alpha_{2 j+1}\right) \rightarrow y_{2}$. Let $H_{j}=\alpha\left(\left[t_{2 j}, t_{2 j+1}\right]\right)$ and $r_{j} \rightarrow 0$ such that $0<r_{j}<\frac{\epsilon}{2}$ and $H_{j} \subset B\left(y, r_{j}\right)$ for every $j \in \mathbf{N}$. Then there exists $\delta>0$ such that $d\left(f\left(H_{j}\right)\right) \geq \delta$ for every $j \in \mathbf{N}$ and $f\left(H_{j}\right)$ is a continua and Card $f\left(H_{j}\right)>1$ for $j \in \mathbf{N}$. Let $M \subset Y$ be a continuum with Card $M>1$ and such that $f\left(D \cap B\left(y, \frac{\epsilon}{2}\right)\right) \subset Y \backslash M$. We have two cases.

Case 1. There exists $y_{0} \in Y$ and $R>0$ and an infinite set $J_{1} \subset \mathbf{N}$ such that $f\left(H_{j}\right) \cup M \subset B\left(y_{0}, R\right)$ for every $j \in J_{1}$. Let $\Gamma_{j}^{\prime}=\Delta\left(f\left(H_{j}\right), M, Y\right)$ for $j \in J_{1}$. Using Theorem A, if $\rho_{1}=C_{1} R^{Q-q-1} \min \{\delta, d(M)\}$ we see that $M_{q}\left(\Gamma_{1}^{\prime}\right) \geq \rho_{1}$ for every $j \in J_{1}$.

Case 2. There exists $y_{0} \in Y, R>0$ and an infinite set $J_{2} \subset N$ such that $M \subset B\left(y_{0}, R\right)$ and $f\left(H_{j}\right) \cap S\left(y_{0}, R\right) \neq \phi, f\left(H_{j}\right) \cap S\left(y_{0}, 2 R\right) \neq \phi, f\left(H_{j}\right) \subset B\left(y_{0}, 3 R\right)$ for $j \in J_{2}$. Let $\rho_{2}=C_{1}(3 R)^{Q-q-1} \min \{R, d(M)\}$. We see from Theorem A that $M_{q}\left(\Gamma_{j}^{\prime}\right) \geq \rho_{2}$ for every $j \in J_{2}$. 
Let $J=J_{1} \cup J_{2}$ and $\rho=\min \left\{\rho_{1}, \rho_{2}\right\}$. We proved that $M_{q}\left(\Gamma_{j}^{\prime}\right) \geq \rho$ for every $j \in J$. Let $\Gamma_{j}$ be the family of all maximal liftings of some paths from $\Gamma_{j}^{\prime}$ starting from some points of $H_{j}$ or $Q_{j}$ for $j \in J$. Let $\Gamma_{1 j}=\left\{\alpha \in \Gamma_{j} \mid \alpha\right.$ has at least a limit point in $\left.\partial D \cap B\left(y, \frac{\epsilon}{2}\right)\right\}$ and $\Gamma_{2 j}=\left\{\alpha \in \Gamma_{j} \mid \operatorname{Im} \alpha \cap S\left(y, \frac{\epsilon}{2}\right) \neq \phi\right\}$ for $j \in J$. Using the compactness of $\bar{B}\left(y, \frac{\epsilon}{2}\right)$ and the openness of the mapping $f$, we see that if $\alpha \in \Gamma_{j}$, then either $\alpha$ has at least a limit point in $\partial D \cap B\left(y, \frac{\epsilon}{2}\right)$ or $\operatorname{Im} \alpha \cap S\left(y, \frac{\epsilon}{2}\right) \neq \phi$. Then $\Gamma_{j}=\Gamma_{1 j} \cup \Gamma_{2 j}, \Gamma_{j}^{\prime}>f\left(\Gamma_{j}\right)$ for $j \in J$ and let $\Delta_{j}=\Gamma_{y, r_{j}, \frac{\epsilon}{2}}$ for $j \in J$. We see that $\Gamma_{2 j}>\Delta_{j}$ for $j \in J$ and we see from Lemma 2.5 that $\lim _{j \rightarrow \infty} M_{\omega}^{p}\left(\Delta_{j}\right)=0$.

Let $\Gamma_{1 j}^{r}=\left\{\gamma \in \Gamma_{1 j} \mid \gamma\right.$ is rectifiable $\}$ for $j \in J$. We see from Theorem 2.3 that $M_{\omega}^{p}\left(\Gamma_{1 j}\right)=M_{\omega}^{p}\left(\Gamma_{1 j}^{r}\right)$ for $j \in J$. Let now $j \in J$ be fixed and let $\alpha:[0,1) \rightarrow X, \alpha \in \Gamma_{1 j}^{r}$. Then there exists $\lim _{t \rightarrow 1} \alpha(t)=a_{\alpha} \in X$ and obviously there exists $\lim _{t \rightarrow 1} f(\alpha(t)) \in \bar{Y}$ and hence $a_{\alpha} \in E$. Now $E \cap B\left(y, \frac{\epsilon}{2}\right) \subset E \cap B(b, \epsilon)$ and $M_{\omega}^{p}(E \cap B(b, \epsilon))=0$ and hence $M_{\omega}^{p}\left(E \cap B\left(y, \frac{\epsilon}{2}\right)\right)=0$ and this implies that $M_{\omega}^{p}\left(\Gamma_{1 j}^{r}\right)=0$ for every $j \in J$. We have

$$
\begin{aligned}
\rho & \leq M_{q}\left(\Gamma_{j}^{\prime}\right) \leq M_{q}\left(f\left(\Gamma_{j}\right)\right)=M_{q}\left(f\left(\Gamma_{1 j}\right) \cup f\left(\Gamma_{2 j}\right)\right) \leq M_{q}\left(f\left(\Gamma_{1 j}\right)\right)+M_{q}\left(f\left(\Gamma_{2 j}\right)\right) \\
& \leq \gamma\left(M_{\omega}^{p}\left(\Gamma_{1 j}\right)\right)+\gamma\left(M_{\omega}^{p}\left(\Gamma_{2 j}\right)\right)=\gamma\left(M_{\omega}^{p}\left(\Gamma_{1 j}^{r}\right)\right)+\gamma\left(M_{\omega}^{p}\left(\Gamma_{2 j}\right)\right) \leq \gamma\left(M_{\omega}^{p}\left(\Delta_{j}\right)\right) \rightarrow 0
\end{aligned}
$$

if $j \rightarrow \infty$. We reached a contradiction and hence $M_{\omega}^{p}(E \cap B(b, \epsilon))>0$ for every $b \in B$ and every $\epsilon>0$. A similar argument holds if $Y$ is compact.

\section{References}

[1] Adamowicz, T.: Prime ends in metric spaces and boundary extensions of mappings. - Anal. Math. Phys. 9, 2019, 1941-1975.

[2] Adamowicz, T., A. Buörn, J. Buörn, and N. Shanmugalingam: Prime ends for domains in metric spaces. - Adv. Math. 238, 2013, 459-505.

[3] Adamowicz, T., and N. Shanmugalingam: Non-conformal Loewner type estimates for modulus of curve families. - Ann. Acad. Sci. Fenn. Math. 35, 2010, 609-626.

[4] Afanas'eva, E.: Boundary behaviour of ring $Q$-homeomorphisms on Riemannian manifolds. - Ukr. Math. J. 63:10, 2012, 1479-1493.

[5] Afanas'eva, E., V. Ryazanov, and R. Salimov: On mappings in the Orlicz-Sobolev classes on Riemannian manifolds. - J. Math. Sci. 181:1, 2012, 1-17.

[6] Balogh, Z., K. Fässler, and K. Peltonen: Uniformly quasiregular maps on the compactified Heisenberg group. - J. Geom. Anal. 22:3, 2012, 633-665.

[7] Balogh, Z., and P. Koskela: Quasiconformality, quasisymmetry and removability in Loewner space. - Duke Math. J. 101:3, 2000, 555-577.

[8] Balogh, Z., P. Koskela, and S. Rogovin: Absolute continuity of quasiconformal mappings on curves. - Geom. Funct. Anal. 17:3, 2007, 645-664.

[9] Cristea, M.: Quasiregularity in metric spaces. - Rev. Roumaine Math. Pures Appl. 51:3, 2006, 607-631.

[10] Cristea, M.: Open, discrete mappings having local $A C L^{n}$ inverses. - Complex Var. Elliptic Equ. 55:1-3, 2010, 61-90.

[11] CRistea, M.: Local homeomorphisms satisfying generalized modular inequalities. - Complex Var. Elliptic Equ. 59:2, 2014, 232-246.

[12] CRistea, M.: Some properties of open, discrete generalized ring mappings. - Complex Var. Elliptic Equ. 61:5, 2016, 623-643.

[13] Cristea, M.: Eliminability results for mappings satisfying generalized modular inequalities. - Complex Var. Elliptic Equ. 64:4, 2019, 676-684. 
[14] Cristea, M.: Exceptional sets for the definition of quasiregularity. - J. Math. Anal. Appl. 485:2, 2020, 123789 .

[15] Golberg, A., and R. Salimov: Logarithmic Hölder continuity of ring homeomorphisms with controlled p-moduli. - Complex Var. Elliptic Equ. 59:1, 2014, 91-98.

[16] Golberg, A., and E. Sevost'yanov: Absolute continuity on path of spatial discrete mappings. - Anal. Math. Phys. 81:1, 2018, 25-35.

[17] Golberg, A., R. Salimov, and E. Sevost'yanov: Singularities of discrete, open mappings with controlled p-moduli. - J. Anal. Math. 127:1, 2015, 303-328.

[18] Golberg, A., R. Salimov, and E. Sevost'yanov: Estimates for Jacobian and dilatation coefficients of open, discrete mappings with controlled $p$-moduli. - Complex Anal. Oper. Theory 11:7, 2017, 1521-1542.

[19] Golberg A., and R. Salimov: Hölder continuity of homeomorphisms with controlled growth of their spherical means. - Complex Anal. Oper. Theory 11:8, 2017, 1825-1838.

[20] Graves, I. M.: The theory of functions of real variables. - McGraw-Hill, 1946.

[21] Guo, C. Y., and M. Williams: The branch set of quasiregular mappings between metric manifolds. - C. R. Acad. Sci. Paris Ser. I 354, 2016, 155-159.

[22] Guo, C. Y., and M. WiLliams: Geometric function theory: The art of pullback factorisation. - arXiv:16110747v1[math.cv], 2016, 1-93.

[23] Heinonen, J.: Lectures on analysis on metric spaces. - Springer Verlag, New York, 2001.

[24] Heinonen, J., and P. Koskela: Definitions of quasiconformality. - Invent. Math. 120, 1995, $61-79$.

[25] Heinonen, J., and P. Koskela: Quasiconformal maps on metric spaces with controlled geometry. - Acta Math. 181, 1998, 1-61.

[26] Heinonen, J., P. Koskela, N. Shanmugalingam, and J. Tyson: Sobolev classes of Banach valued functions and quasiconformal mappings. - J. Anal. Math. 85, 2001, 87-139.

[27] Hencl, S., and P. Koskela: Lectures on mappings of finite distortion. - Lecture Notes in Math. 2096, Springer, 2014.

[28] Hurewicz, W., and H. Wallman: Dimension theory. - Princeton Univ. Press, 1948.

[29] Ignat'ev, A., and V. RYazanov: Finite mean oscillation in mapping theory. - Ukr. Math. Bull. 2, 2005, 403-424.

[30] Il'yutko, D., and E. Sevost'yanov: Boundary behaviour of open, discrete mapping on Riemannian manifolds. - Sb. Math. 209:5, 2018, 605-651.

[31] Iwaniec, T., and G. Martin: Geometric function theory and non-linear analysis. - Oxford Math. Monogr., The Clarendon Press, Oxford Univ. Press, New York, 2001.

[32] Korány, A., and H. Reimann: Quasiconformal mappings on the Heisenberg group. - Invent. Math. 80:2, 1985, 309-338.

[33] Korány, A., and H. Reimann: Foundations for the theory of quasiconformal mappings on the Heisenberg group. - Adv. Math. 111:1, 1995, 1-87.

[34] Koskela, P., and J. Onninen: Mappings of finite distortion: Capacity and modulus inequalities. - J. Reine Angew. Math. 599, 2005, 1-26.

[35] Kovtonyuk, D., V. Ryazanov, and R. Salimov: Towards the theory of Orlicz-Sobolev classes. - St. Petersburg Math. J. 25:6, 2014, 929-963.

[36] Martio, O., S. Rickman, and J. VÄIsÄLÄ: Topological and metric properties of quasiregular mappings. - Ann. Acad. Sci. Fenn. Ser. A I Math. 488, 1971, 1-31.

[37] Martio, O., S. Rickman, and J. VäısÄLÄ: Definitions for quasiregular mappings. - Ann. Acad. Sci. Fenn. Ser. A I 448, 1969, 1-40. 
[38] Martio, O., and S. Rickman: Boundary behaviour of quasiregular mappings. - Ann. Acad. Sci. Fenn. Ser. A I Math. 507, 1978, 1-17.

[39] Martio, O., V. Ryazanov, U. Srebro, and E. Yakubov: Mappings of finite length distortion. - J. Anal. Math. 93, 2004, 215-236.

[40] Martio, O., V. Ryazanov, U. Srebro, and E. Yakubov: On $Q$-hemeomorphisms. - Ann. Acad. Sci. Fenn. Math. 30:1, 2005, 49-69.

[41] Martio, O., V. Ryazanov, U. Srebro, and E. Yakubov: Moduli in modern mapping theory. - Springer Monogr. Math., Springer, New York, 2009.

[42] Noshiro, K.: Cluster sets. - Springer Verlag, Berlin, 1960.

[43] Onninen, J., and K. RaJala: Quasiregular maps to generalized manifolds. - J. Anal. Math. $109,2009,33-79$.

[44] Rajala, K.: Mappings of finite distortion: Removable singularities for locally homeomorphic mappings. - Proc. Amer. Math. Soc. 123, 2004, 3251-3258.

[45] Reshetnyak, Y.G.: Space mappings of bounded distortion. - Transl. Math. Monogr. 73, Providence, RI, Amer. Math. Soc., 1989.

[46] Rickman, S.: Quasiregular mappings. - Ergeb. Math. Grenzgeb. (3) 26, Berlin, Springer Verlag, 1993.

[47] Ryazanov, V., R. Salimov, and E. Sevost'yanov: On convergence analysis of space homeomorphisms. - Siberian Adv. Math. 23:4, 2013, 263-293.

[48] Ryazanov, V., and E. Sevost'yanov: Towards the theory of ring Q-homeomorphisms. Israel J. Math. 168, 2008, 101-115.

[49] Ryazanov, V., and E. Sevost'yanov: Equicontinuity of mappings quasiconformal in the mean. - Ann. Acad. Sci. Fenn. Math. 36, 2011, 1-14.

[50] RYazanov, V., and S. Volkov: On the boundary behaviour of mappings in the class $W_{\text {loc }}^{1,1}$ on Riemann surfaces. - Complex Anal. Oper. Theory 11, 2017, 1503-1520.

[51] Ryazanov, V., and S. Volkov: Prime ends in the Sobolev mappings on Riemann surfaces. - Mat. Stud. 48, 2017, 24-36.

[52] Salimov, R.: On regular homeomorphisms in the plane. - Ann. Acad. Sci. Fenn. Math. 35, 2010, 285-289.

[53] Salimov, R.: On the Lipschitz property of a class of mappings. - Mat. Zametki 94:4, 2013, $591-599$.

[54] Salimov, R., and E. Sevost'yanov: $A C L$ and differentiability of the open, discrete ring mappings. - Complex Var. Elliptic Equ. 55:1-3, 2010, 49-59.

[55] Salimov, R., and E. Sevost'yanov: The Poletskii and Väisälä inequalities for the mappings with $(p, q)$ distortion. - Complex Var. Elliptic Equ. 59:2, 2014, 217-231.

[56] Sevost'yanov, E.: Towards a theory of removable singularities for maps with unbounded characteristics of quasi-conformality. - Izv. Math. 74:1, 2010, 151-165.

[57] Sevost'yanov, E.: On the branch points of mappings with unbounded coefficient of quasiconformality. - Sib. Math. J. 51:5, 2010, 899-912.

[58] Sevost'yanov, E.: Local and boundary behaviour of maps in metric spaces. - St. Petersburg Math. J. 28:6, 2017, 807-824.

[59] Sevost'yanov, E.: On boundary extension of mappings in metric spaces in terms of primes ends. - Ann. Acad. Sci. Fenn. Math. 44, 2019, 65-90.

[60] Sevost'yanov, E.: On the local and boundary behaviour of mappings of factor spaces. Complex Var. Elliptic Equ., doi: 10.1080/17476933.2020.1825392.

[61] Sevost'yanov, E., and A. Markysh: On Sohotski-Casorati-Weierstrass theorem on metric spaces. - Complex Var. Elliptic Equ. 64:12, 2019, 1973-1993. 
[62] Sevost'yanov, E., and S. Skvortshov: On the convergence of mappings in metric spaces with direct inverse modulus conditions. - Ukr. Math. J. 70:7, 2018, 1097-1114.

[63] Sevost'yanov, E., and S. Skvortsov: On mappings whose inverses satisfy the Poletsky inequality. - Ann. Acad. Sci. Fenn. Math. 45, 2020, 259-277.

[64] SmolovayA, E.: Boundary behaviour of ring $Q$-homeomorphisms in metric measure spaces. Ukr. Math. J. 62:5, 2010, 785-793.

[65] VÄISÄLÄ, J.: Lectures on $n$-dimensional quasiconformal mappings. - Lecture Notes in Math. 229, Springer Verlag, Berlin, 1971.

[66] Vuorinen, M.: Conformal geometry and quasiregular mappings. - Lecture Notes in Math. 1319, Springer-Verlag, Berlin, 1988.

[67] Whyburn, G. T.: Analytic topology. - Colloquium Publications 28, Amer. Math. Soc., 1942.

[68] Wilson, D.: Open mappings on manifolds and a counter example to the Whyburn conjecture. - Duke Math. J. 40, 1973, 703-716.

Received 25 August 2020 • Revised received 5 February 2021 • Accepted 12 March 2021 